\title{
Synthesis, Spectral Characterization and Molecular Docking Studies of Novel Benzidin-bis-tetrahydroacridine Analogues
}

\author{
MADALINA MARINA HRUBARU ${ }^{*}$, CARMELLINA DANIELA BADICEANU², \\ ANTHONY CHINONSO EKENNIA ${ }^{3 *}$, SUNDAY N. OKAFOR ${ }^{4}$, CRISTIAN ENACHE $^{5}$, \\ LASZLO TARKO ${ }^{1}$, VICTORITA TECUCEANU ${ }^{1}$, MARIA MAGANU ${ }^{1}$ \\ ${ }^{1}$ Romanian Academy, Organic Chemistry Center "C.D.Nenitescu", 202B Splaiul Independentei Str., 060023, Bucharest, \\ Romania \\ ${ }^{2}$ University of Medicine and Pharmacy, Carol Davila, Faculty of Farmacy, Department of Pharmaceutical Chemistry, 6 Traian \\ Vuia Str., 020956, Bucharest, Romania \\ ${ }^{3}$ Department of Chemistry, Alex Ekwueme Federal University Ndufu-Alike (AE-FUNAI), P.M.B 1010 Abakiliki, Ebonyi State, \\ Nigeria \\ ${ }^{4}$ Department of Pharmaceutical and Medicinal Chemistry, University of Nigeria, Nsukka 410001, Enugu State, Nigeria \\ ${ }^{5}$ National Phytosanitary Laboratory, National Phytosanitary Authority, 11 Voluntari Blvd., 077910, Voluntari, Romania
}

Abstract. Alzheimer's is a progresive neurodegenerative disease that interferes with human cognitive ability, memory and behavior. The inhibition of acetylcholinesterase (AChE) and butyrylcholinesterase $(B C h E)$ enzymes are major therapeutic routes for the treatment of Alzheimer disease. In the study, nevel bis-polymethylenquinoline-bis-carboxamides (3a-f) and bis-polymethylenquinoline-bis-carboxylic acids (5a-b) having as precursor benzidine, were obtained in good yields by Pfitzinger condensation reactions of bis-isatines with corresponding cyclanones. The compounds were characterized by elemental analysis, FT-IR, NMR and mass spectrometry. Furthermore, the compounds were subjected to molecular docking dynamics simulations to ascertain their potentials as inhibitors of acetylcholinesterase (AChE) and butyrylcholinesterase (BChE). Molecular docking simulations showed varied binding activities towards the two binding sites of acetylcholinesterase: 4EY7 and 1OCD, and human butyrylcholinesterase: 1P0I. Compounds $3 e$ and $5 b$ demostrated strong binding affinities with 1P0I, 1OCD and 4EY7 biotargets similar to the binding modes of donepezil and tacrine (co-crystallized inhibitors of acetylcholinesterase) and butyrate (co-crystallized inhibitors of butyrylcholinesterase).

Keywords:bis-tetrahydroacridin-9-carboxamides; bis-tetrahydroacridin-9-carboxylic acid; bis-polymethylenquinoline-bis-carboxamides;acetylcholinesterase inhibitors (AChEI); butyrylcholinesterase inhibitors (BChEI); Alzheimer's disease

\section{Introduction}

Alzheimer's disease (AD) [1] is a progressive physical disorder that causes increasingly severe impairment in the cognitive and functional ability of individuals suffering from the disease [2] and finally leads to dementia [3]. It has been established that AD disease has a multifactorial pathoetiological origin [4], which implicate hallmarks like deposits of aberrant proteins ( $\beta$-amyloid and $\tau$-protein) [6], oxidative stress [7], dyshomeostasis of biometals [8], and low levels of acetylcholine (ACh) [9]. Trying to explain the Alzheimer's disease several hypothesis have been issued. The first hypothesis proposed, which still underpins the current available drug therapies for $\mathrm{AD}$, is the cholinergic hypothesis. According to this hypothesis, $\mathrm{AD}$ is caused by the decrease in the number of cholinergic neurons and reduced synthesis of the neurotransmitter acetylcholine (ACh) [10] especially in the hippocampus and cortex, leading to cognitive and memory deficits. One way to improve neuronal transmission is to reduce or inhibit the activity of the two cholinestereases involved in the degradation of the neurotransmitter:

*email: madalina.hrubaru@gmail.com, chemisttony@gmail.com 
acetylcholinesterase (AChE, E.C.3.1.1.7) and butyrylcholinesterase (BuChE, E.C.3.1.1.8). The inhibition of acetylcholinesterase (AChE) and butyrylcholinesterase (BChE) enzymes are major therapeutic routes for the treatment of Alzheimer disease.

The structure of the two enzymes is well known: they contain a catalytic active site (CAS) located at the bottom of a long and narrow gorge and a peripheral anionic site (PAS) located at the entry to the active gorge [11]. Acetylcholinesterase (AChE) is the main enzyme involved in the hydrolysis of the Ach, while butyrylcholinesterase (BuChE) acts as a coregulator of the activity of AChE, particularly in the brain areas associated with learning and memory [12]. During progression of AD, the activity of AChE decreases while that of BuChE register a progressive and significant increase, influencing cognition, awareness, and behavior by modulating ACh levels in the central nervous system (CNS) [13]. Acetylcholinesterase (AChE), also plays a proaggregating (non-catalytic) role, accelerating b-amyloid peptide (A $\beta$ ) aggregation and deposition into the neurofibrillary tangles [14], in which the peripheral anionic binding site (PAS) of AChE is involved [15]. AChE inhibitors (AChEIs) are the most effective class of medications for short term (6 to 12 months) treatment of $\mathrm{AD}$, which improves cognitive function and physical activities, with generally favorable tolerability and safety profiles [16].

Administration of cholinesterase inhibitors can modify the progress of AD by decreasing the activity of AChE, and maintaining higher ACh levels in the cleft, as well as enhanced transmission of the electric impulses. The presence of an amide group (-NHCO-) and polyalkyquinolinic skeleton in the structure of tetrahydroacridines or their bioisosteres is a common scaffold found in an important number of potential acetylcholinesterase inhibitors [17]. An example is 9-aminotetrahydroacridine (tacrine) which is a very potent $\mathrm{AChE}$ and $\mathrm{BuChE}$ inhibitor (more potent toward the latter enzyme), which increases the levels of ACh by stimulation of muscarinic receptors [18]. It was the first drug approved for the treatment of AD, but was later withdrawn [19] due to its hepatotoxicity [20]. The mechanisms of action of tetrahydroacridines are based on their interaction with the residues located at CAS and mid-gorge recognition sites (Figure 2A) of AChE. Over the decades intense synthetic and pharmacological studies were dedicated to design more effective and less aggressive AChE inhibitors and many of the studies have been focused on developing new substrates, containing the tacrine skeletal [21]. Because of the multifactorial nature of $\mathrm{AD}$ and the numerous cerebral mechanisms implicated in the control of $\mathrm{AD}$, the standard one molecule-one target solution [22] has proved not to be very effective and was abandoned in favor for a "multi-target-directed strategy". In the attempt to obtain molecules that react simultaneously with multiple targets in the complex of the neuronal cascades, the tacrine moiety again has been widely used in the development of the new multitarget compounds (MTDLs) for Alzheimer's disease treatment. Many of the ChEs inhibitors (bis-tacrines derivatives and hybrids of tacrine with other moieties) were synthesized by connecting different substituted tacrines with identical or different moiety, through an aminoalkyl spacer of diverse length and fixed functionality. The new tetrahydroacridine multi-targeted analogues show dual or multiple biological profiles and demonstrate better efficacy [23], such as: A $\beta$ aggregation [20], AChE/BuChE and beta secretase inhibitory properties, inhibitors of cholinesterase and monoamine oxidases, multifunctional compounds with metal-chelating properties, multifunctional compounds with nitric-oxide releasing properties, anti-inflammatory properties or with tau protein hyperphosphorilation inhibition properties [24].

As a contribution to the development of new compounds with AChE/BuChE inhibitory potentials, we report here the synthesis and molecular docking studies of new bis-tetrahydroacridine analogues, based on benzidine, in which the two tetrahydroacridine units are connected directly through the benzenic nucleus of tetrahydroacridinic skeleton (Figure1). 

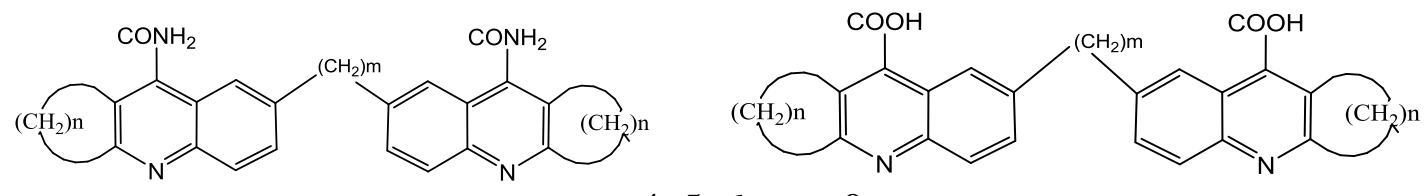

$\mathrm{n}=4,5,6 ; \mathrm{m}=0$

Figure 1. Tetrahydroacridinic skeleton of the novel dual inhibitors

Previously, we described the synthesis of other tetrahydroacridine derivatives [25,26] obtained by Pfitzinger reaction of isatines with cyclanones and their AChE inhibitory activity was predicted using the structure-based QSAR model recently developed in our lab [27]. The QSAR model indicated a correlation between the higher number of methylene groups present in the target molecule and the higher inhibitory AChE activity. In the present work, novel eight bis tetrahydroacridines compounds were synthesized and subjected to molecular docking studies and their binding energies, Drug-likeness and Pharmacokinetics evaluations were carried out.

\section{Materials and methods}

All chemicals were purchased from commercial suppliers and used without any further purification. The IR spectra were recorded using a FT-IR Bruker Vertex 70 equipped with a diamond ATR. ${ }^{1} \mathrm{H}$ and ${ }^{13} \mathrm{C}$ NMR spectra were recorded using a Gemini 300BB spectrometer operating at $300 \mathrm{MHz}$ for ${ }^{1} \mathrm{H}$ and $75 \mathrm{MHz}$ for ${ }^{13} \mathrm{C}$ in DMSO- $\mathrm{d}_{6}$, using TMS as internal reference. Mass spectra were recorded on Varian 1200 LC/MS/MS mass spectrometer with electrospray interface ESI, both in positive and negative mode. Substance dissolved in methanol (1\% formic acid) to $1 \mathrm{ppm}$ was direct introduced ESI interface using a Prostar 240 SDM pump at $50 \mu \mathrm{l}$ flow rate. ESI needle was set at $5000 \mathrm{~V}$ both positive and negative mode, drying and nebulizing gas was nitrogen from Domnick Hunter generator. For negative mode methanol had $0.5 \%$ ammonium carbonate instead of formic acid. Fragmentations were performed in second quadrupole using argon as collision gas at 1.5 mTorr. Fragments were scanned by the third quadrupole.

\subsection{Synthesis}

\subsubsection{Synthesis of precursors}

a. Procedure for the synthesis of bis-isonitrosoacetanilide (N,N'-(biphenyl-4,4'-diyl)bis(2-(hydroxyimino)acetamide) (1a)

Chloral hydrate $(0.22 \mathrm{~mol}, 32.5 \mathrm{~g})$ was dissolved in $1000 \mathrm{~mL}$ water with $\mathrm{Na}_{2} \mathrm{SO}_{4}(1.60 \mathrm{~mol}, 230 \mathrm{~g})$. The solution was warmed at $60^{\circ} \mathrm{C}$ before the solution of hydroxylamine hydrochloride $(0.22 \mathrm{~mol}$ in $100 \mathrm{~mL}$ water) was added. In a separate flask, slurry of benzidine $(0.1 \mathrm{~mol}, 18.4 \mathrm{~g})$ in water $(120 \mathrm{~mL})$ with concentrated $\mathrm{HCl}(17.3 \mathrm{~mL})$ was prepared. The two reaction mixtures were combined and the reaction temperature was increased to $90-95^{\circ} \mathrm{C}$ for $20 \mathrm{~min}$. Heating was then stopped and the mixture was cooled in an ice bath for $15 \mathrm{~min}$. The solid precipitates were collected by filtration, recrystalized in $\mathrm{C}_{2} \mathrm{H}_{5} \mathrm{OH}$, filtrated off, and poured into a cold dilute ammonia solution. The resultant yellowish precipitates (Figure 2) were obtained by filtration, washed with water $(30 \mathrm{~mL})$ and $\mathrm{C}_{2} \mathrm{H}_{5} \mathrm{OH}$, and dried [28].

Yield: 39\% (12.71g); Mp: over $240^{\circ} \mathrm{C}$; Anal.calcd for $\mathrm{C}_{17} \mathrm{H}_{16} \mathrm{~N}_{4} \mathrm{O}_{4}(326.1)$ : C 62.61, H 4.96, N 17.19. Found: C 63.25, H4.99, N 17.21; FT-IR(solid, ATR, $\mathrm{cm}^{-1}$ ): 3317m, 3135m, 3042m, 2998m, 2867w , 1635vs, 1620vs , 1590s ; ${ }^{1} \mathrm{H}-\mathrm{NMR}$ (DMSO-d $6, \delta$ ppm, $J$ Hz): 12.22 (sl, 2H, H-10, H-10'), 10.30(s, 2H, H7, H-7'), 7.77 (d, J=8.8, 4H, H-3, H-5, H-3', H-5'), 7.68 (s, 2H, H-9, H-9'), 7.63(d, J=8.8, 4H, H-2, H-6, H-2', H-6'); ${ }^{13}$ C-NMR (DMSO-d $6, \delta$ ppm): 160.28 (C-8, C-8'), 144.12 (C-9, C-9'), 137.67 (C-1, C-1'), 135.05 (C-4, C-4'), 126.06(C-3, C-5, C-3', C-5'), 120.26(C-2, C-6, C-2', C-6'). ESI-MS : (m/z) 325 ([M- 


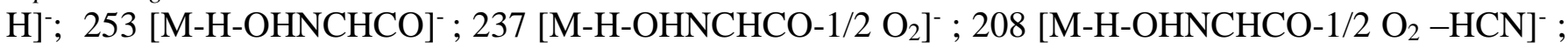
208 [M-H-OHNCHCO-1/2 $\left.\mathrm{O}_{2}-\mathrm{HCN}-\mathrm{CO}\right]^{-}$.

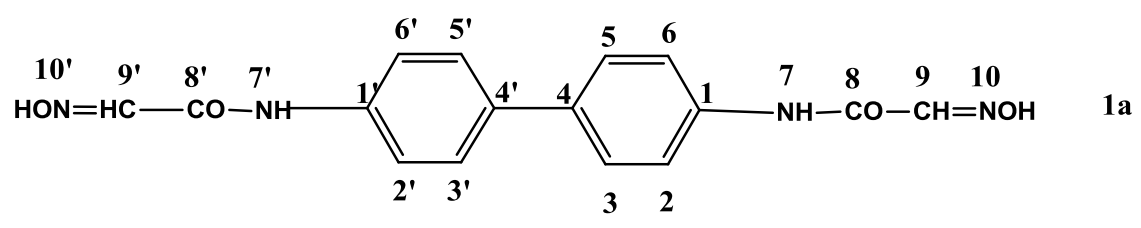

Figure 2. $N, N^{\prime}$-(biphenyl-4,4'-diyl)bis(2-(hydroxyimino)acetamide (1a)

b. General procedure for the synthesis of bis-indanone $(2 a)$

$N, N^{\prime}$-(biphenyl-4,4'-diyl)bis(2-(hydroxyimino)acetamide derivative (1a) $(0.03 \mathrm{~mol}, 9.78 \mathrm{~g})$ is added in small portions, over 8-10 minutes, to a solution of sulphuric acid $(0.72 \mathrm{~mol}, 44.14 \mathrm{~mL}, 88.3 \%, \mathrm{~d}=1.81)$ at $70-72^{\circ} \mathrm{C}$, under vigorous stirring. The temperature is maintained at $87-95^{\circ} \mathrm{C}$ for 30 min to complete the reaction. The mixture is cooled at room temperature and poured into a mixture of ice and water. The crude product is stirred for $30 \mathrm{~min}$, filtered off and washed several times with water to neutral $p \mathrm{H}$. Deep-purple powder was obtained (Figure 3 ). The bis-isatines $(2 a)$ were purified by dissolving it in an aqueous alkali $5 \%$, neutralising with dilute acetic acid, filtering off the dark material separated, and acidifying the solution with hydrochloric acid [29]. The solution is then cooled rapidly, and the bis-isatin which separates is filtered off.

Yield 61\% (5.35g). Mp: over $240^{\circ} \mathrm{C}$; Anal.calcd. $\mathrm{C}_{16} \mathrm{H}_{8} \mathrm{~N}_{2} \mathrm{O}_{4}$ (292.25): C 65.88, H 2.76, N 9.59. Found: C 65.88, H 2.83, N 9.31; FT-IR(solid, ATR, $\mathrm{cm}^{-1}$ ): 1737s, 1708s, 1616m, 1510m de adaugat inca cateva valori; ${ }^{1} \mathrm{H}-\mathrm{NMR}\left(\mathrm{DMSO}_{6}, \delta \mathrm{ppm}, J \mathrm{~Hz}\right): 11.14(\mathrm{~s}, 2 \mathrm{H}, \mathrm{H}-1, \mathrm{H}-1$ ') $)$ 7. 89(dd, J=8.2, J=1.9; 2H, H6, H-6'); 7.75(d, $J=1.9,2 \mathrm{H}, \mathrm{H}-4, \mathrm{H}-4$ '); 6.99(d, $J=8.2,2 \mathrm{H}, \mathrm{H}-7, \mathrm{H}-7$ '); ${ }^{13} \mathrm{C}-\mathrm{NMR}$ (DMSO-d $6, \delta$ ppm): 184.44(C-3, C-3'); 159.65(C-2, C-2'); 149.84(C-7a, C-7a'); 136.19(C-4,C-4'); 133.64(C-5,C-5'); 122.28(C-6, C-6'); 118.51(C-4a, C-4'a); 112.88(C-7, C-7'). MS ESI-MS negative mode ( $\mathrm{m} / \mathrm{z}): 291$ [M-H] $^{-}$ ; $263[\mathrm{M}-\mathrm{H}-\mathrm{CO}]^{-} ; 235[\mathrm{M}-\mathrm{H}-2 \mathrm{CO}]^{-} ; 220\left[\mathrm{M}-\mathrm{H}-2 \mathrm{CO}-1 / 2 \mathrm{~N}_{2} \mathrm{H}_{2}\right]^{-}$.

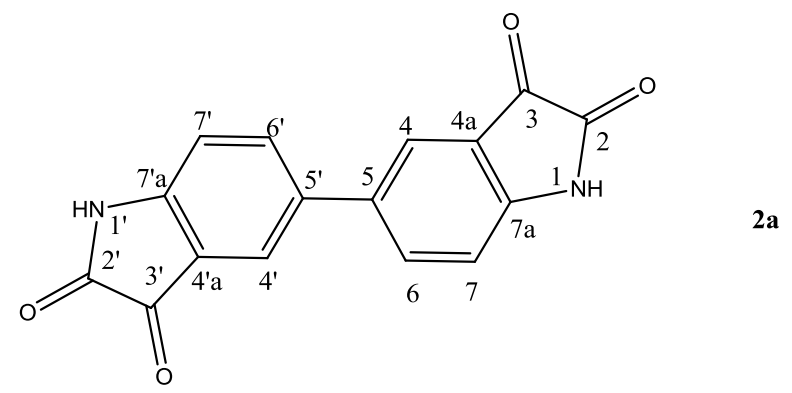

Figure 3. 5,5'-biindoline-2,2',3,3'-tetraone (2a)

\subsubsection{General procedure for synthesis of carboxamides $(3 a-f)$}

Target compounds (3a-f) were obtained according the reaction outlined in scheme 1 by cyclocondensation of bis-indoline-tetraones with a cyclanone: 5,5'-biindoline-2,2',3,3'-tetraone ( 0.0033 mol, $0.96 \mathrm{~g})(2 a)$, cyclanone $(0.0098 \mathrm{~mol})$ and $5 \mathrm{~mL}$ aqueous ammonia $25 \%$ were heated in an autoclave at $150^{\circ} \mathrm{C}$ for $2 \mathrm{~h}$. The crude product was cooled, washed with acetone-water 1:1, boiled in chloroform and filtered off. All the condensation products were high melting substances, above $250^{\circ} \mathrm{C}$ with decomposition, insoluble in common organic solvents and so could not be purified as such. 
5, $5^{\prime}, 6,6^{\prime}, 7,7^{\prime}, 8,8^{\prime}$-octahydro-2,2'-biacridine-9,9'-dicarboxamide (3a).

Following the general procedure, 5,5'-biindoline-2,2',3,3'-tetraone (0.0033 mol, 0.96g) (2a), cyclohexanone $(0.0098 \mathrm{~mol}, 0.96 \mathrm{~g})$ and $5 \mathrm{~mL}$ aqueous ammonia $25 \%$ were used.

Yeld 79\% (1.17g); Mp: over $240^{\circ} \mathrm{C}$; Anal.calcd : $\mathrm{C}_{28} \mathrm{H}_{28} \mathrm{~N}_{4} \mathrm{O}_{2}(450) ; \mathrm{N}$ 12.44. Found:12.19; IR(solid, ATR, $\mathrm{cm}^{-1}$ ): 3335m, 3064m, 2947m, 2867m, 2827m, 1655vs, 1615s, 1565s, 1488w, 1391w, 1121w, 816w, 626w; ' ${ }^{\mathrm{H}-\mathrm{NMR}}$ (DMSO-d 6 +TFA, $\delta$ ppm, $J$ Hz): 8.57, 8.52(sl, 4H, NH, H-11, H-11' ); 8.46(dd, $\left.J=8.4 ; J=1,9,2 \mathrm{H}, \mathrm{H}-3, \mathrm{H}-3^{\prime}\right) ; 8.38\left(\mathrm{~d}, J=8.4,2 \mathrm{H}, \mathrm{H}-4, \mathrm{H}-4^{\prime}\right) ; 8.20(\mathrm{~d}, J=1.9,2 \mathrm{H}, \mathrm{H}-1, \mathrm{H}-1$ '); $3.41(\mathrm{sl}, 4 \mathrm{H}$, H-5, H-5'); 3.02 (sl, 4H, H-8, H-8'); 1.8-2.01(m, 8H, H-6, H-6',H-7, H-7'); ${ }^{13} \mathrm{C}-\mathrm{NMR}$ (DMSO-d 6 +TFA, $\delta$ ppm): 166.13(C-11, C-11'); 150.26(C-9, C-9'); 139.17(C-5a, C-5'a); 137.10(C-4a, C-4'a); 133.22(C-1, C-1'); 129.16(C-8a, C-8'a); 124.02(C-4, C-4'); 123.87(C-1a, C-1'a); 122.27(C-3, C-3'); $121.08(\mathrm{C}-2$, C2'); 29.42(C-8, C-8'); 25.64(C-5, C-5'); 21.03(C-7, C-7'); 20.60(C-6, C-6'); ESI-MS positive mode: 451 $[\mathrm{M}+\mathrm{H}]^{+} ; 406\left[\mathrm{M}+\mathrm{H}-\mathrm{CO}-\mathrm{NH}_{3}\right]^{+} ; 361\left[\mathrm{M}+\mathrm{H}-2 \mathrm{CO}-2 \mathrm{NH}_{3}\right]^{+} ; 226[\mathrm{M}+2 \mathrm{H}]^{2+}$.

7,7'-dimethyl-5, 5',6,6',7,7',8,8'-octahydro-2,2'-biacridine-9,9'-dicarboxamide) (3b).

Following the general procedure, 5,5'-biindoline-2,2',3,3'-tetraone ( $0.0033 \mathrm{~mol}, 0.96 \mathrm{~g})(2 a)$, 4 methylcyclohexanone $(0.0098 \mathrm{~mol}, 1.009 \mathrm{~g})$ and $5 \mathrm{~mL}$ aqueous ammonia $(25 \%)$ were used.

Yeld $81 \%$ (1.28g); Mp: over $240^{\circ} \mathrm{C}$; Anal.calcd: $\mathrm{C}_{30} \mathrm{H}_{30} \mathrm{~N}_{4} \mathrm{O}_{2}(478.6)$ : C 75.29; H, 6.32; N 11.72 . Found: C 75.38, H 6.82, N 11.84; IR(solid, ATR, $\mathrm{cm}^{-1}$ ): 3379m, 3193w, 2928w, 2861w, 1648s, $1459 \mathrm{w}$, 1399 w, 1361w, 1318w, 1112w, 883w; ${ }^{1} \mathrm{H}-\mathrm{NMR}$ ( DMSO- d 6 , $\delta$ ppm, $J$ Hz): 8.57; 8.52 (sl, 4H, NH, H-11, H-11' ); 8.45(dd, J=9.7, $J=1.9,2 \mathrm{H}, \mathrm{H}-3, \mathrm{H}-3$ ' ); 8.37(d, $J=9.7,2 \mathrm{H}, \mathrm{H}-4, \mathrm{H}-4$ '); 8.23(d, $J=1.9,2 \mathrm{H}, \mathrm{H}-1, \mathrm{H}-$ 1'); 3.13(sl, 4H, H-5, H-5'); 3.08(sl, 4H, H-8, H-8'); 1.6-2.01(m, 6H, H-6, H-6'; H-7, H-7'); 1.11(d, $\left.J=6.3, \quad 6 \mathrm{H}, \mathrm{H}-12, \mathrm{H}-12{ }^{\prime}\right) ;{ }^{13} \mathrm{C}-\mathrm{NMR}$ (DMSO-d $6, \delta \mathrm{ppm}$ ): 168.55(C-11, C-11'); $162.90\left(\mathrm{C}-9, \mathrm{C}^{\prime} 9^{\prime}\right)$; 145.23(C-5a, C-5'a); 142.47(C-4a, C-4'a); 137.15(C-8a, C-8'a); 129.54(C-4, C-4' ); 127.91(C-3, C-3'); 125.80(C-1a, C-1'a); $122.90\left(\mathrm{C}-2, \mathrm{C}^{\prime}{ }^{\prime}\right)$; $122.23(\mathrm{C}-1, \mathrm{C}-1$ '); 36.47(C-12, C-12'); 30.63(C-8; C-8'); 26.52(C-5; C-5'); 20.95(C-7; C-7'), 19.82(C-6; C-6'); ESI-MS: $479[\mathrm{M}+\mathrm{H}]^{+} ; 463\left[\mathrm{M}+\mathrm{H}_{-} \mathrm{CH}_{4}\right]^{+} ; 447$ $\left[\mathrm{M}+\mathrm{H}-2 \mathrm{CH}_{4}\right]^{+} ; 404\left[\mathrm{M}+\mathrm{H}-2 \mathrm{CH}_{4}-\mathrm{HNCO}\right]^{+} ; 240[\mathrm{M}+2 \mathrm{H}]^{2+}$.

5,5'-dimethyl-5, 5',6,6',7,7',8, 8'-octahydro-2,2'-biacridine-9,9'-dicarboxamide (3c) .

Following the general procedure, 1g 5,5'-biindoline-2,2',3,3'-tetraone $(2 a)(0.0033 \mathrm{~mol}, 0.96 \mathrm{~g}), 2$ methylcyclohexanone $(0.0098 \mathrm{~mol}, 1.098 \mathrm{~g})$ and $5 \mathrm{~mL}$ aqueous ammonia, $25 \%$ were used.

Yield 77\% (1.21g). Mp: over $240^{\circ} \mathrm{C}$ Anal.calcd. $\mathrm{C}_{30} \mathrm{H}_{30} \mathrm{~N}_{4} \mathrm{O}_{2}$ (478.6): C 75.29, H 6.32, N 11.72. Found: C 75.38, H 6.47, N 11.81. IR(solid, ATR, $\mathrm{cm}^{-1}$ ): 3350m, 3033, 2928m, 2868w, 1653vs, 1618s, $1565 \mathrm{~m}, 1490 \mathrm{~m}, 1431 \mathrm{~m}, 1397 \mathrm{~m}, 1364 \mathrm{w}, 1330 \mathrm{w}, 1210 \mathrm{w}, 821 \mathrm{w}, 624 \mathrm{w} .{ }^{1} \mathrm{H}-\mathrm{NMR}$ (DMSO-d $6, \delta \mathrm{ppm}, J \mathrm{~Hz}$ ): 8.25; 8.11 (s, 4H, NH, H-11, H-11'); 8.09 (d, J=8.5, 2H, H-4, H-4'); 8.05 (d, J=1.7, 2H, H-1, H-1' ); 8.02 (d, J=8.5, J=1.7, 2H, H-3, H-3'); 3.15(m , 2H, H-5, H-5'); 2.97(t, J=6.4, 4H, H-8, H-8'); 1.47(d, J=6.9, $6 \mathrm{H}, \mathrm{H}-12, \mathrm{H}-12{ }^{\prime}$ ) ; 1.6-2.2(m, 8H, H-6, H-6', H-7, H-7'); ${ }^{13} \mathrm{C}-\mathrm{NMR}$ (DMSO-d $6, \delta$ ppm): 168.60(C-11, C11'); 162.94(C-9, C-9'); 145.27(C-5a, C-5'a); 142.50(C-4a, C-4'a); 137.20(C-2, C-2'); 129.59(C-4, C-4'); 127.98(C-3, C-3'); 125.87(C-8a, C-8'a); 122.95(C-1a, C-1'a); 122.28(C-1, C-1'); 36.51(C-5, C-5'); 30.67(C-6, C-7, C-6', C-7' ); 26.57(C-8, C-8'); 20.99(C-12, C-12'); 19.86(C-7, C-6, C-7', C-6'). ESI-MS positive mode: $479[\mathrm{M}+\mathrm{H}]^{+} ; 463\left[\mathrm{M}+\mathrm{H}-\mathrm{CH}_{4}\right]^{+} ; 447\left[\mathrm{M}+\mathrm{H}-2 \mathrm{CH}_{4}\right]^{+} ; 434\left[\mathrm{M}+\mathrm{H}-2 \mathrm{CH}_{4}-\mathrm{NH}_{3}\right]^{+} ; 240$ $[\mathrm{M}+2 \mathrm{H}]^{2+}$.

7,7',8,8',9,9',10,10'-octahydro-6H,6'H-2,2'-bi(cyclohepta[b]quinoline)-11,11'-dicarboxamide (3d).

Following the general procedure, 5,5'-biindoline-2,2',3,3'-tetraone $(2 a)(0.0033 \mathrm{~mol}, 0.96 \mathrm{~g})$, cycloheptanone $(0.0098 \mathrm{~mol}, 1.099 \mathrm{~g})$ and $5 \mathrm{~mL}$ aqueous ammonia, $25 \%$ were used. 
Yield 77\% (1.22g). Mp: over $240^{\circ} \mathrm{C}$ Anal.calcd.: $\mathrm{C}_{30} \mathrm{H}_{30} \mathrm{~N}_{4} \mathrm{O}_{2}$ (478.6); C 75.29, H 6.32, N 11.72. Found: C 75.65, H 11.94, N 11.89: IR(solid, ATR, $\mathrm{cm}^{-1}$ ): 3319s, 3021, 2920s, 2911, 2851,1655s, 1623s 1573, 1491,1401, 1182, 1127, 958, 824. ${ }^{1} \mathrm{H}-\mathrm{NMR}$ (DMSO-d,$\delta$ ppm, $J \mathrm{~Hz}$ ): 8.26; 8.09 (s, 4H, H-11, H$\left.11^{\prime}\right) ; 8.07$ (d, J=8.7, 2H, H-4, H-4'); 8.01(dd, 2H, J=8.7, 1.9; H-3, H-3' );7.97(d, 2H, J=1.9, H-1, H-1'); 3.21(m, 4H, H-10, H-10'); 2.95(m, 4H, H-6, H-6'); 1.86(tl, 4H, H-7, H-7' ); 1.76(sl, 4H, H-8, H-8'); 1.74(m, 4H, H-9, H-9'); ${ }^{13} \mathrm{C}-\mathrm{NMR}$ (DMSO-d $6, \delta$ ppm, $J$ Hz): 168.86(C-12, C-12'); 164.99(C-11, C-11'); 144.79(C-4a, C-4'a, C-6a, C-6'a); 141.94(C-6a, C-6'a, C-4a, C-4'a), 137.48( C-2, C-2'), 131.42(C-10a, C-10'a); 129.44(C-3, C-3'); 127.97(C-1, C-1'); 123.52(C-1a, C-1'a); 122.66(C-4, C-4'); 31.27(C-9, C-9', C-5 , C-5’); 30.54(C-6 ,C-6', C-10, C-10'); 27.69(C-7,C-7’, C-8, C-8' ); 26.64(C-9, C-9'); ESI-MS positive mode: $479[\mathrm{M}+\mathrm{H}]^{+} ; 434\left[\mathrm{M}+\mathrm{H}-\mathrm{CO}-\mathrm{NH}_{3}\right]^{+} ; 240[\mathrm{M}+2 \mathrm{H}]^{2+}$.

6,6',7,7',8,8',9,9',10,10',11,11'-dodecahydro-2,2'-bi(cycloocta[b]quinoline)-12,12'-dicarboxamide (3e).

Following the general procedure, $1 \mathrm{~g}$ 5,5'-biindoline-2,2',3,3'-tetraone (2a) (0.0033 mol, 0.96g), cycloctanone $(0.0098 \mathrm{~mol}, 1.24 \mathrm{~g})$ and $5 \mathrm{~mL}$ aqueous ammonia, $25 \%$ were used.

Yield 71\% (1.19g). Mp: over $240^{\circ} \mathrm{C}$ Anal.calcd.: $\mathrm{C}_{32} \mathrm{H}_{34} \mathrm{~N}_{4} \mathrm{O}_{2}$ (506): C 75.86, H 6.76, N 11.07. Found: C 78.98, H 6.95, N 11.37: IR(solid, ATR, $\mathrm{cm}^{-1}$ ): 3337, 2970, 2925, 2852, 2788, 1663,1619, 1565, 1481, 1431, 1332, 1128, 825; ${ }^{1} \mathrm{H}-\mathrm{NMR}$ (DMSO-d $6, \delta$ ppm, $J$ Hz): 8.31; 8.28 (s, 4H, NH, H-13, H-13'); 8.10 (d, $J=8.8,2 \mathrm{H}, \mathrm{H}-4, \mathrm{H}-4$ '); 8.04 (dd, $J=8.8, J=1.9,2 \mathrm{H}, \mathrm{H}-3, \mathrm{H}-3$ '); 7.98 (d, J=1.9, 2H, H-1, H-1'); 3.11(sl, 4H, H-11, H-11'); 3.0(sl, 4H, H-6, H-6'); 1.8 (sl, 8H, H-7, H-7', H-10, H-10'); 1.4 (sl, 8H, H-8, H-9, H8', H-9'); ${ }^{13} \mathrm{C}-\mathrm{NMR}$ (DMSO-d 6 , $\delta$, ppm): 168.88 (C-13, C-13'), 163.65 (C-12, C-12'); 145.49(C-2, C-2'); 142.84 (C-6a, C-6'a); 137.31 (C-4a, C-4'a); 129.63 (C-4, C-4'); 129.46(C-3, C-3'); 127.96 (C-1a (C11a)); 123.38 (C-11a (C-1a)); 122.61(C-1, C-1');37.58 (C-11,C-11';C-6, C-6’); 34.96 ((C-6(C-6'); C-11 $\left.\left(\mathrm{C}-11^{\prime}\right)\right)$; 30.84(C-10(C-10'), C-7(C-7')); 28.03(C-10(C-10’), C-7(C-7')), 25.61(C-8(C-8'), C-9(C-9')); ESI-MS positive mode: $507[\mathrm{M}+\mathrm{H}]^{+} ; 490\left[\mathrm{M}+\mathrm{H}-\mathrm{NH}_{3}\right]^{+} ; 434\left[\mathrm{M}+\mathrm{H}-\mathrm{NH}_{3}-2 \mathrm{CO}\right]^{+} ; 254[\mathrm{M}+2 \mathrm{H}]^{2}$.

\section{5,5',6,6'-tetrahydro-9,9'-bibenzo[c]acridine-7,7'-dicarboxamide (3f).}

Following the general procedure, $1 \mathrm{~g}$ 5,5'-biindoline-2,2',3,3'-tetraone, $2 a$ (0.0033 mol, 0.96g), tetralone $(0.0098 \mathrm{~mol}, 1.43 \mathrm{~g})$ and $5 \mathrm{~mL}$ aqueous ammonia , $25 \%$ were used.

Yield $69 \%$ (1.24g). Mp: over $240^{\circ} \mathrm{C}$ Anal.calcd.: $\mathrm{C}_{36} \mathrm{H}_{26} \mathrm{~N}_{4} \mathrm{O}_{2}(546)$ : C 79.10; $\mathrm{H} 4.79 ; \mathrm{N} 10.25$. Found: C 78.13; H 4.89; N 10.91; IR(solid, ATR, $\mathrm{cm}^{-1}$ ): 3315m, 3066, 2929, 2845, 1624s, 1492, 1433, 1390, 1361,1238 ,1158, 826, 703, 468; ' H-NMR (DMSO-d $6, \delta$ ppm, $J$ Hz) : 8.48(m, 2H, H-1, H-1'); 8.36(2H, NH, H-13, H-13'); 8.25(d, J=9.4, 2H, H-11, H-11'); 8.18(s, 2H, NH, H-13, H-13' ); 8.14(dd, J=9.4, $J=1.4,2 \mathrm{H}, \mathrm{H}-10, \mathrm{H}-10$ '); 8.10(d, J=1.4, 2H, H-8, H-8'); 7.35-7.50(m,6H, H-2, H-3, H-4, H-2', H-3', H-4' ); 2.95-3.20(m, 8H, H-5, H-6, H-5', H-6'); ${ }^{13} \mathrm{C}-\mathrm{NMR}$ ( DMSO-d 6 , $\delta$ ppm): 168.48(C-13,C-13'); $163.02(\mathrm{C}-$ 13, C-13'); 152.78(C-7, C-7'); 146.36 (C-11a(C-11'a), C-12a (C-12'a)); 142.23 (C-12a (C-12'a) ,C$11 \mathrm{a}(\mathrm{C}-11$ 'a)); 139.68 (C-9, C-9'); 137.94(C-8a (C-6a), C-8'a(C-6'a)); 134.10 (C-8'a(C-6'a),C-8a(C-6a)); 130.58 (C-2,C-2',C-11,C-11'); 128.50 (C-4,C-4'), 128.76 (C-8,C-8'); 127.48 (C-3,C-3'); 126.43 (C-4a, C4'a); 125.98 (C-1,C-1'); 124.17 (C-1a, C-1'a); 122.90 (C-10, C-10'); 27.36 (C-6(C-5), C-6'(C-5')), 25.90 (C-5 (C-6), C-5'(C-6')); Positive ESI-MS: $547[\mathrm{M}+\mathrm{H}]^{+} ; 502\left[\mathrm{M}+\mathrm{H}-\mathrm{CO}-\mathrm{NH}_{3}\right]^{+} ; 485\left[\mathrm{M}+\mathrm{H}-\mathrm{CO}-2 \mathrm{NH}_{3}\right]^{+}$; $457\left[\mathrm{M}+\mathrm{H}-2 \mathrm{CO}-2 \mathrm{NH}_{3}\right]^{+}$.

\subsubsection{General procedure for synthesis of corresponding dicarboxylic acids (5a-b):}

Target compounds $(5 a-b)$ were obtained according the reaction oulined in scheme 1 , by cyclocondensation of bis-indoline-tetraones with a cyclanone in etanolic solution of $\mathrm{KOH}$ : 0.1 mol of benzidinbisisatin ( $2 a$ ) was dissolved in $0.3 \mathrm{~mol}$ of $\mathrm{KOH}$ (30\% solution) in $100 \mathrm{~mL}$ of EtOH. At the end, $0.3 \mathrm{~mol}$ of cyclanone is added with stirring. The reaction was stirred at reflux for 12 hours. Then the half 
of the solution is concentratad by vacuum distilation and the precipitate formed is filtered by vacuum and washed several times with acetone and ethyl ether. After cooling 0.34-0.4 moles of 50\% $\mathrm{CH}_{3} \mathrm{COOH}$ were added. The resulting yellow solid was insoluble in common organic solvents and therefore could not be purified. The compounds decompose above $240^{\circ} \mathrm{C}$.

2,2',3,3'-tetrahydro-1H,1'H-7,7'-bi(cyclopenta[b]quinoline)-9,9'-dicarboxylic acid (5a).

Following the general procedure, $0.1 \mathrm{~mol}$ of benzidinbisisatin $(2 a), \mathrm{KOH}(0.3 \mathrm{~mol}, 16.83 \mathrm{~g})(30 \%$ solution), cyclopentanone ( $0.3 \mathrm{~mol}, 25.24 \mathrm{~g} \mathrm{~g})$, gave $14.85 \mathrm{~g}$ of the title compound.

Yield 35\% (14.85g); Mp: over $240^{\circ} \mathrm{C}$ Anal.calcd. $\mathrm{C}_{26} \mathrm{H}_{20} \mathrm{~N}_{2} \mathrm{O}_{4}$ (424.46): C 73.57, H 7.75, N, 6.60. Found: C 73.11, H 7.89, N 6.75; IR(solid, ATR, $\mathrm{cm}^{-1}$ ): 3300m, 2923m, 2878m, 1692fi, 1615, 1494fi, $1517 \mathrm{~s}, 1454 \mathrm{~s}, 1214 \mathrm{~m}, 812 \mathrm{~m} ;{ }^{1} \mathrm{H}-\mathrm{NMR}\left(\mathrm{CD}_{3} \mathrm{NO} 2-\mathrm{d}_{6}{ }^{+} \mathrm{TFA}, \delta \mathrm{ppm}, J \mathrm{~Hz}\right): 10.9(\mathrm{~s}, 2 \mathrm{H}, \mathrm{NH}, \mathrm{H}-10$ ', H-10); 10.6(s, 2H, NH, H-10', H-10); 9.00 (d, $J=1.9,2 \mathrm{H}, \mathrm{H}-8, \mathrm{H}-8^{\prime}$ ) ; 8.55 (dd, 2H, $J=8.9, J=1.9, \mathrm{H}-6, \mathrm{H}-6$ '); 8.43 (d, 2H, J=8.9, H-5, H-5'); 3.69 (t, 4H, J=7.4, H-3, H-3') ; 3.54(t, 4H, J=7.4, H-1, H-1'); 2.58 (cv, 4H, $\left.J=7.4, \mathrm{H}-2, \mathrm{H}-2^{\prime}\right) ;{ }^{13} \mathrm{C}-\mathrm{NMR}\left(\mathrm{CD}_{3} \mathrm{NO}_{2}-\mathrm{d}_{6}{ }^{+} \mathrm{TFA}, \delta \mathrm{ppm}\right): 169.35(\mathrm{C}-10, \mathrm{C}-10 ') ; 166.76(\mathrm{C}-9$; C-9'); 142.46(C-5a, C-5'a); 141.83(C-7, C-7'); 141.55(C-3a, C-3'a); 138.29(C-8,C-8'); 134.25(C-8a, C-8'a); 126.47(C-1a, C-1'a); 126.31(C-6, C-6'); 122.66(C-5,C-5'); 33.63(C-1, C-1'); 32.07(C-3, C-3'); 24.15(C2,C-2'); ESI-MS negative mode: 423 [M-H] ${ }^{-} ; 379\left[\mathrm{M}^{\prime} \mathrm{H}-\mathrm{CO}_{2}\right]^{-} ; 335\left[\mathrm{M}-\mathrm{H}-2 \mathrm{CO}_{2}\right]^{-}$.

\section{5,5',6,6',7,7',8, 8'-octahydro-2,2'-biacridine-9,9'-dicarboxylic acid (5b).}

Following the general procedure, $0.1 \mathrm{~mol}$ of benzidinbisisatin $(2 a), \mathrm{KOH}(0.3 \mathrm{~mol}, 16.83 \mathrm{~g})(30 \%$ solution), cyclohexanone ( $0.3 \mathrm{~mol}, 29.44 \mathrm{~g})$, gave $18.55 \mathrm{~g}$ of the title compound.

Yield 45\%; Mp: over $240^{\circ} \mathrm{C}$ Anal.calcd. $\mathrm{C}_{28} \mathrm{H}_{24} \mathrm{~N}_{2} \mathrm{O}_{4}$ (452.51): C 74.32, H 5.35, N 6.19. Found: C 73.98, H 5.84, N 6.45; IR(solid, ATR, $\mathrm{cm}^{-1}$ ): 3405i, 3301i, 3206m, 2944, 2869, 1589i, 1392m, 1205m, 738w, 684w. ${ }^{1} \mathrm{H}-\mathrm{NMR}$ (DMSO-d ${ }^{+}{ }^{+}$TFA, $\delta$ ppm, $J$ Hz) $: 8.12$ (d, $J=8.8,2 \mathrm{H}, \mathrm{H}-4, \mathrm{H}-4$ ' ) $) 8.03$ (dd, $J=1.7$, $\left.J=8.8,2 \mathrm{H}, \mathrm{H}-3, \mathrm{H}^{\prime} 3^{\prime}\right) ; 7.57\left(\mathrm{~d}, J=1.7,2 \mathrm{H}, \mathrm{H}-1, \mathrm{H}^{\prime}{ }^{\prime}\right.$ ) ; 3.29(t, $J=3.32$, 2H,H-5, H-5'); $2.91(\mathrm{t}, J=6.32,2 \mathrm{H}, \mathrm{H}-8$, $\left.\mathrm{H}-8^{\prime}\right) ;{ }^{13} \mathrm{C}-\mathrm{NMR}$ ( DMSO- ${ }_{6}^{+}$TFA, $\delta$ ppm): 168.51(C-11); 159.38(C-11'); $145.17\left(\mathrm{C}-5 \mathrm{a}, \mathrm{C}-8^{\prime} \mathrm{a}\right)$; 142.55(C-4a, C-4'a); 137.18(C-2, C-2'); $129.28\left(\mathrm{C}-1^{\prime}, \mathrm{C}^{\prime} 1^{\prime}\right) ; 128.14\left(\mathrm{C}-4, \mathrm{C}^{\prime} 4^{\prime}\right) ; 126.17\left(\mathrm{C}-3, \mathrm{C}^{\prime} 3^{\prime}\right)$; 123.072(C-1a, C-1'a); 122.38(C-8a, C-8'a); 33.51(C-8, C-8'); 26.14(C-5, C-5'); 22.45(C-7, C-7'); 22.17(C-6, C-6'). ESI-MS positive mode: $453[\mathrm{M}+\mathrm{H}]^{+} ; 409\left[\mathrm{M}+\mathrm{H}-\mathrm{CO}_{2}\right]^{+} ; 363\left[\mathrm{M}+\mathrm{H}-\mathrm{CO}-\mathrm{H}_{2} \mathrm{O}-\right.$ $\left.\mathrm{CO}_{2}\right]^{+}$. ESI-MS negaitive mode: $451[\mathrm{M}-\mathrm{H}]^{-} ; 363\left[\mathrm{M}-\mathrm{H}-2 \mathrm{CO}_{2}\right] ; 225[\mathrm{M}-2 \mathrm{H}]^{2-}$.

\subsection{Molecular docking studies}

Acetylcholinesterase with different inhibitors were used in this study. They are recombinant human acetylcholinesterase (PDB Code: 4EY7) complexed with 1-Benzyl-4-[(5,6-dimethoxy-1-indanon-2yl)methyl]piperidine (donepezil) as an inhibitor, Torpedo Californica acetylcholinesterase (E.C. 3.1.1.7) (PDB Code: 1ODC) complexed with N-4'-Quinolyl-N'-9"-(1",2",3",4"-tetrahydroacridinyl)-1,8diaminooctane (tacrine) as an inhibitor, and human butyryl cholinesterase (PDB Code: 1P0I) complexed with butanoic acid as an inhibitor. Together with their co-crystallized ligands, the 3D crystal structures of 4EY7 at resolution of $2.3 \AA$ (44), 1ODC at resolution of $2.2 \AA$ (45) and 1POI at resolution of $2.0 \AA$ [30] were retrieved from protein data bank repository (https://www.rcsb.org/). They were opened in Discovery Studio and multiple chains and water of crystallization were removed. The synthesized compounds were drawn using Accelrys Draw 4.1. Both the prepared proteins and compounds were energy minimized using MMFF94x force field. The energy minimized compounds were docked into the binding cavities of the proteins. The binding free energy for each compound against the target was calculated. 


\subsubsection{Drug-likeness and Pharmacokinetics Studies}

The physicochemical properties to determine the drug-likeness of the synthesized compounds were evaluated in silico. The properties evaluated include molecular weight (MW); hydrogen bond acceptor (HBA); hydrogen bond donor (HBD); total polar surface area (TPSA); number of rotatable bond (nRB); number of acid (nAc); molar refractivity (MR); number of atoms (nA). Also, the pharmacokinetic parameters were generated using Pre ADMET online free software (www.preadmet.bmdrc.kr). The pharmacokinetic parameters evaluated include: cell membrane permeability (Caco2), human intestinal absorption (HIA), CYP 2C19 inhibition, blood-brain barrier (BBB) and plasma protein binding (PPB).

\section{Results and discussions}

\subsection{Chemistry}

The general synthetic procedure for the new heterodimers is shown in Scheme 1, and consists of three steps:

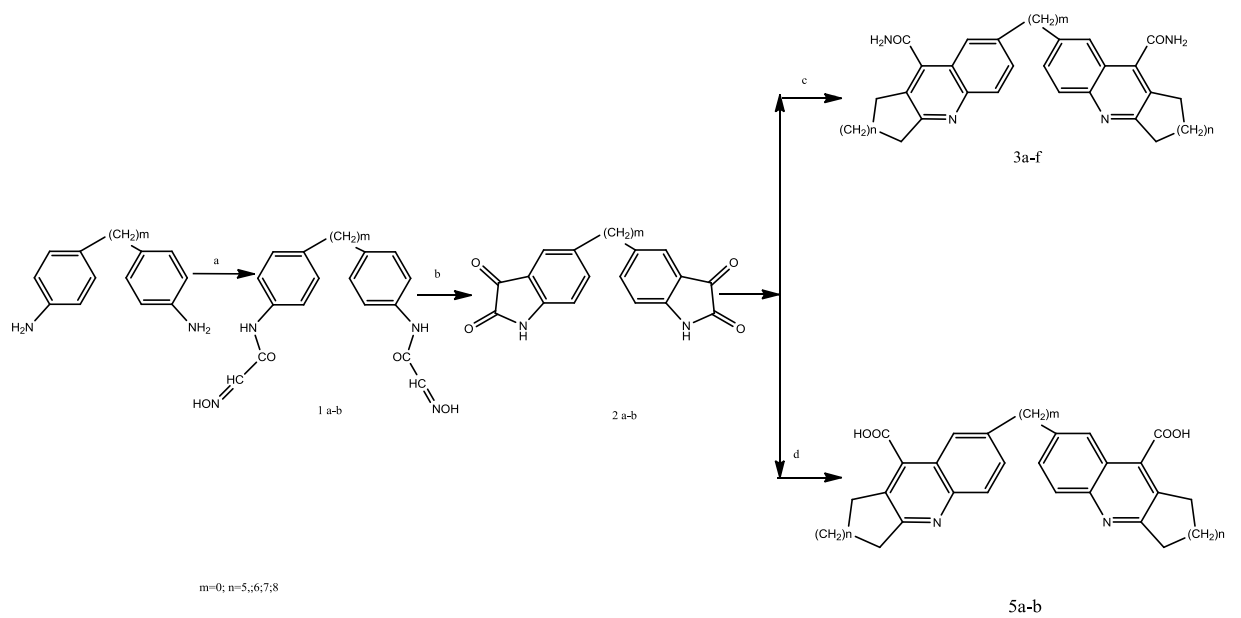

Scheme 1. Synthesis of tetrahydroacridines analogous

a. $\mathrm{CCl}_{3}-\mathrm{CHO}, \mathrm{NH}_{2} \mathrm{OH}, \mathrm{Na}_{2} \mathrm{SO}_{4}, \mathrm{t}=95-100{ }^{\circ} \mathrm{C} ; \mathrm{b} . \mathrm{H}_{2} \mathrm{SO}_{4}, \mathrm{t}=70^{\circ} \mathrm{C} ; \mathrm{c} . \mathrm{NH}_{3}, \mathrm{Cyclanones}$;

d. $\mathrm{KOH}$ alcoholic solution, Cyclanones (used cyclanones were: cyclohexanone,

2-methyl-cyclohexanone, 4-methylcyclohexanone, cycloheptanone, cyclooctanone, tetralone, indanone)

The bis-isonitrosoacetanilides, la were prepared in low yield (30\%) by Sandmayer reaction of dianiline with chloral and hydroxylamine. In the second step of the synthesis, compound $(1 a)$ were heated at $87-95^{\circ} \mathrm{C}$ in $\mathrm{H}_{2} \mathrm{SO}_{4}$ for twenty minutes to obtain the corresponding bisindoline-tetraones (2a), in good yields, by cyclocondensation. In the third step, carboxamides are obtained by Pfitzinger condensation of bis-isatine (2a) with cyclanones in aqueous ammonia on heating $\left(130-150^{\circ} \mathrm{C}\right)$, in a closed vessel. If the Pfitzinger condensation occurred in solution of $\mathrm{KOH}$ in ethanol, the corresponding acids are obtained. The final compounds have little solubility in organic solvents and melting points above $240{ }^{\circ} \mathrm{C}$ with decomposition $[28,29]$. The new compounds were boiled several times in chloroform in order to remove the soluble impurities as no purification of the di-amides $(3 a-f)$ and di-acides $(5 a-b)$, was achieved by recristalization, and/or classic chromatography.

Finally semipreparative HPLC Chromatography is done, with a reversed phase Zorbax C18 column $(250 \mathrm{~mm} 21.2 \mathrm{~mm}$ i.d.; particle size $8 \mu \mathrm{m}$ ) (DuPont, USA). Mobile phase consisted in 20/40/40 water (pH 8.2 with ammonium carbonate)/ methanol ( $0.5 \%$ ammonium carbonate)/ acetonitrile ( $0.5 \%$ ammonium 
Revista de Chimie

https://revistadechimie.ro

https:/ldoi.org/10.37358/Rev. Chim.1949

carbonate). Flow rate was established at $10 \mathrm{~mL} / \mathrm{min}$, using a Prostar $240 \mathrm{SDM}$ pump (Varian). For chromatography it was used 'heart cut' strategy with a Prostar 330 UV-VIS DAD. Sample dissolved in DMF (1\% formic acid) at $100 \mathrm{mg} / \mathrm{mL}$, was manually injected into system at portion of $1 \mathrm{ml}$, using an Reodyne 7725i . Chromatography was ten times repeated. Solvents (methanol, acetonitrile) were removed in vacuum, from water a grey-beige deposit was collected, filtered off and dried. The method was applied to all synthesized compounds (Figure 4 and 5) [31].
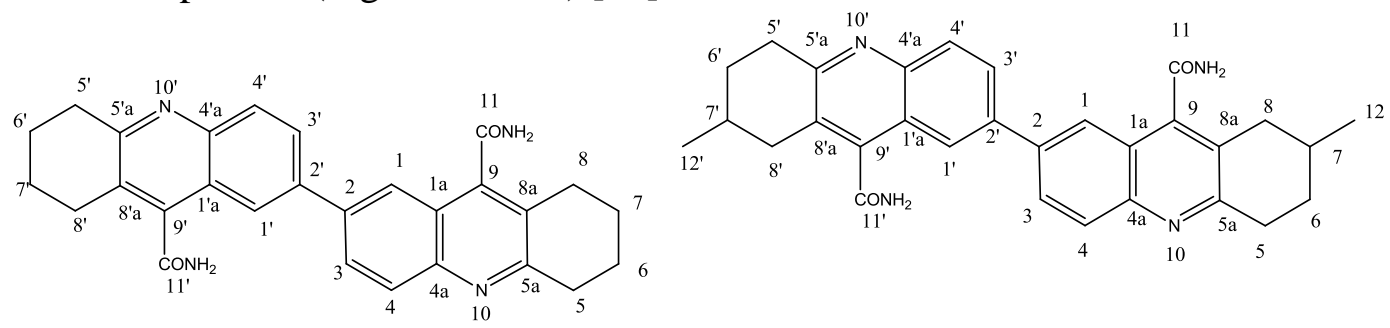

$5,5^{\prime}, 6,6^{\prime}, 7,7^{\prime}, 8,8^{\prime}$-octahydro-2,2'-biacridine-9,9'-dicarboxamide $(3 a)$

7,7'-dimethyl-5,5',6,6',7,7',8,8'-octahydro-[2,2'-biacridine]-9,9'-dicarboxamide $3 \boldsymbol{b}$
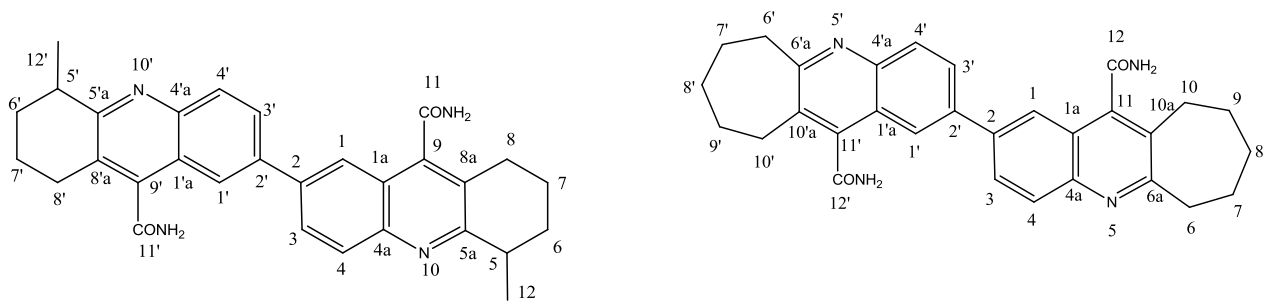

5,5'-dimethyl-5,5',6,6',7,7',8,8'-octahydro-2,2'-biacridine-9,9'-dicarboxamide (3c)

7,7',8,8',9,9',10,10'-octahydro-6H,6'H-2,2'-bi(cyclohepta[b]quinoline)-11,11'-dicarboxamide (3d).
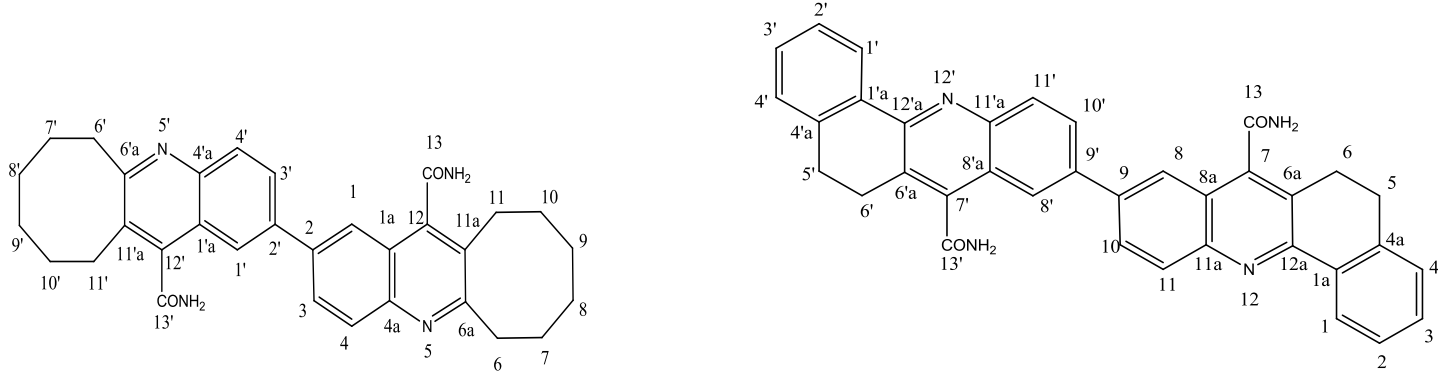

$6,6^{\prime}, 7,7^{\prime}, 8,8^{\prime}, 9,9^{\prime}, 10,10^{\prime}, 11,11^{\prime}$-dodecahydro-2,2'-bi(cycloocta[b]quinoline)-12,12'-dicarboxamide (3e)

$5,5^{\prime}, 6,6^{\prime}$-tetrahydro-[9,9'-bibenzo[c]acridine $]-7,7^{\prime}$-dicarboxamide $3 f$

Figure 4. Structure of carboxamides $(3 a-f)$

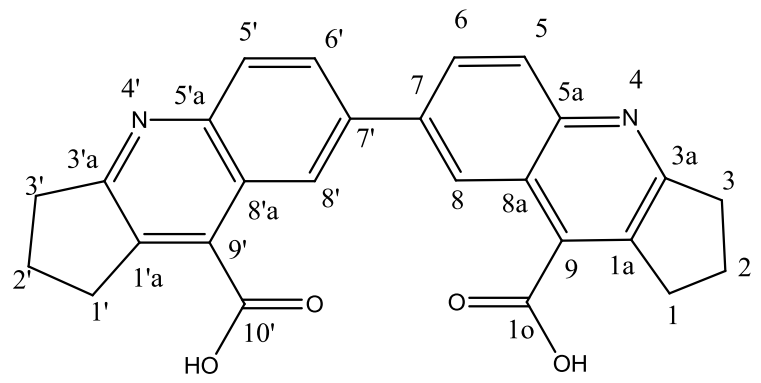

2,2',3,3'-tetrahydro-1H,1'H-7,7'-bi(cyclopenta[b]quinoline)-9,9'-dicarboxylic acid (5a) 


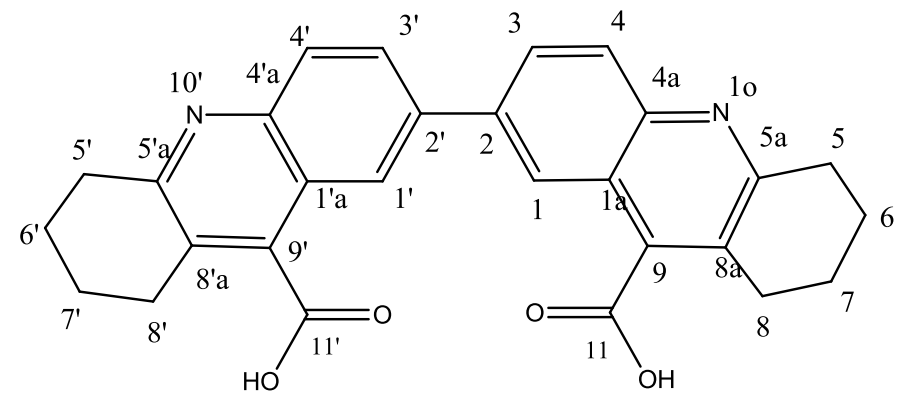

$5,5^{\prime}, 6,6^{\prime}, 7,7^{\prime}, 8,8^{\prime}$-octahydro-2,2'-biacridine-9,9'-dicarboxylic acid $(5 b)$

Figure 5. Structures of dicarboxylic acids $(5 a-b)$

\subsection{NMR Spectral data}

The ${ }^{1} \mathrm{H}$ NMR spectra of isonitrosoacetanilides, $1 a$ shows for the aromatic protons the AA'BB 'systems with coupling constants of $J=8.8 \mathrm{~Hz}$ at $\delta=7.63 ; 7.7 \mathrm{ppm}$. The azomethynic protons occur at about $\delta=$ $7.68 \mathrm{ppm}$. The signals for the amide group proton -NH-CO- appears at $\delta=10.3 \mathrm{ppm}$ and for the hydroxylamine at $\delta=12 \mathrm{ppm}$.

The ${ }^{1} \mathrm{H}$ NMR spectra of bis-isatine, $2 a$ (obtained by acid cyclization) show three distinct signals for each proton in part. The most shielded is the proton $\mathrm{H}-7$ which appears at $\delta=6.9 \mathrm{ppm}$ as a doublet with a coupling constant of $J=8.2 \mathrm{~Hz}$. Proton at H-6 gives a doublet of doublets that occurs at $\delta=7.89 \mathrm{ppm}$. The proton $\mathrm{H}-4$ appears at $\delta=7.75 \mathrm{ppm}$, with a meta coupling constant of $J=1.9 \mathrm{~Hz}$.

By cyclocondensation of bis-isatin, $2 a$ with cyclanones to bis-tacrincarboxamides / bis-tacrinacids, aromatic protons retain their multiplicity, but their chemical shifts are significantly modified in the tetrahydroacridinic system. A deshield of about $\delta=0.8-1 \mathrm{ppm}$ is observed for the proton $\mathrm{H}-7$ (doublet) positioned in the ortho position towards the quinolinic nitrogen atom, and to a lesser extent of about $\delta=$ 0.2 - 0.4 ppm for the proton $\mathrm{H}-4$, of the benzidine. The amide group as well as the carboxyl group, induces a deshielding through space over the $\mathrm{H}-4$ proton and the methylene groups of the saturated cycles which occurs in the range of $\delta=3.01-3.32 \mathrm{ppm}$.

The ${ }^{13} \mathrm{C}$-NMR spectra (see supplementary information) of the isonitrosoacetanilides present signals for $\mathrm{C}-8, \mathrm{C}-8^{\prime}$ carbon atoms of the amidic group - $\mathrm{CO}-\mathrm{NH}$ - groups, at $\delta=160.28 \mathrm{ppm} 1 \mathrm{a}$. The signals for the azomethylic group appear at $\delta=144.12 \mathrm{ppm}(1 a)$. The ${ }^{13} \mathrm{C}$-NMR spectra of bis-isatin (2a) showed signals at $\delta=184,44 \mathrm{ppm}, 2 a$ for C-3, C-3' carbon atoms of the carbonyl groups and for the amide group at $\delta=159 ., 2 a$. The signals for C-7, C-7 ' tertiary carbon atoms occurred at $\delta=112.88$.

The ${ }^{13} \mathrm{C}$ NMR spectra of the bistacrines (resulting from the condensation of the bisisatine $(2 a)$ with different cyclanones) showed signals for the amide group at $\delta=168.5-168.0 \mathrm{ppm}$ and for the acids at $\delta=$ 166.5 - $168.0 \mathrm{ppm}$. The carbon atom in the positions: $\delta, \mathrm{C}-9$ and C-9 'show signals at $\delta=157-162 \mathrm{ppm}$. Compared to bisisatins, tertiary carbon atoms do not suffer significant displacements. The methylene groups of the saturated cycles of bistacrinamides, present signals at $\delta=25-30 \mathrm{ppm}$, the most distorted ones being those placed nearest by the amide and the carboxyl groups.

\subsection{FT-IR Spectra}

The IR (solid ATR) spectra highlight the characteristic functional groups and skeleton frequencies. The amidic groups, both in precursors and in tetrahydroacridinic carboxamides structures, show for $\mathrm{NH}$ group, a stretching vibration $v_{\mathrm{NH}} \in$ in the field $3100-3350 \mathrm{~cm}^{-1}$ and a deformation vibration $\delta_{\mathrm{NH}} \in 1530$ - 
$1580 \mathrm{~cm}^{-1}$. For carbonyl and azomethynic groups, vibrations within the range $v_{\mathrm{CO}} \in 1600-1680 \mathrm{~cm}^{-1}$ were observed.

The FT-IR spectra of the bis tetrahidroacridinic analogous show the vibrations of aromatic $\mathrm{C}=\mid \mathrm{C}$ double bonds in the range $3001-3050 \mathrm{~cm}^{-1}$ and the $v_{\mathrm{C}=\mathrm{N}}$ vibration in the range $1585-1621 \mathrm{~cm}^{-1}$. The vibrations for methylene groups $-\mathrm{CH}_{2}$ - were seen at $2840-2880 \mathrm{~cm}^{-1}$ and $2960-2980 \mathrm{~cm}^{-1}$. Deformation vibrations are found in the range $1340-1465 \mathrm{~cm}^{-1}$, and the deformation vibration of aromatic hydrogens in the range $680-860 \mathrm{~cm}^{-1}$.

\subsection{Mass Spectra}

The basicity of tetrahydroacridines is comparable or even greater than that of the quinoline.

Anyway, in the molecule of tetrahydroacridinic carboxamides, the quinolinic nitrogen has a higher basicity comparing to the amidic nitrogen, due to the presence of the saturated cycle adjacent to the quinolinic N-heterocycle, which increases both the density of electrons and the steric hindrance.

Therefore, electrospray ionization, in the positive ion mode, will produce protonated pseudomolecular ions by the placement of a proton on the nitrogen of the quinolinic heterocycle. When an acidified solvent (e.g. with formic acid) is used for dissolution, the electrospray ionization of the diamides and di-acids, in the positive ion mode, take place with the formation of the parent molecular ion as a protonated molecule $[\mathrm{M}+\mathrm{H}]^{+}$. Instead, when a basic mobile phase (water with $0.5 \%$ ammonium carbonate with a $p \mathrm{H}$ of 8.2) is used, the retention is better for amides and the shape of the chromatographic peaks is significantly improved. Unfortunately, the acids didn't produce good retention neither in acidic nor basic eluents.

Ionization of the amides, Figure 6, occurs with the formation of double ions $[\mathrm{M}+2 \mathrm{H}]^{2+}$ having $\mathrm{m} / \mathrm{z}$ values corresponding to half; the abundance of these ions is significantly much lower than that of the molecular ions $[\mathrm{M}+\mathrm{H}]^{+}$.

Their fragmentation occurs with the successive loss of the amidic groups. In the positive mode, amides are broken down as follows: loss of an ammonia molecule from an amide group, loss of another ammonia molecule from the second amide group, loss of a carbon monoxide molecule (CO), loss of another molecule of carbon monoxide (CO) from the second amide group. Amides without bridge are difficult fragmented.

In the positive mode, amides are broken down as follows: loss of an ammonia molecule from an amide group, loss of another ammonia molecule from the second amide group, loss of a carbon monoxide molecule (CO), loss of another molecule of carbon monoxide (CO) from the second amide group. Amides without bridge are difficult fragmented:

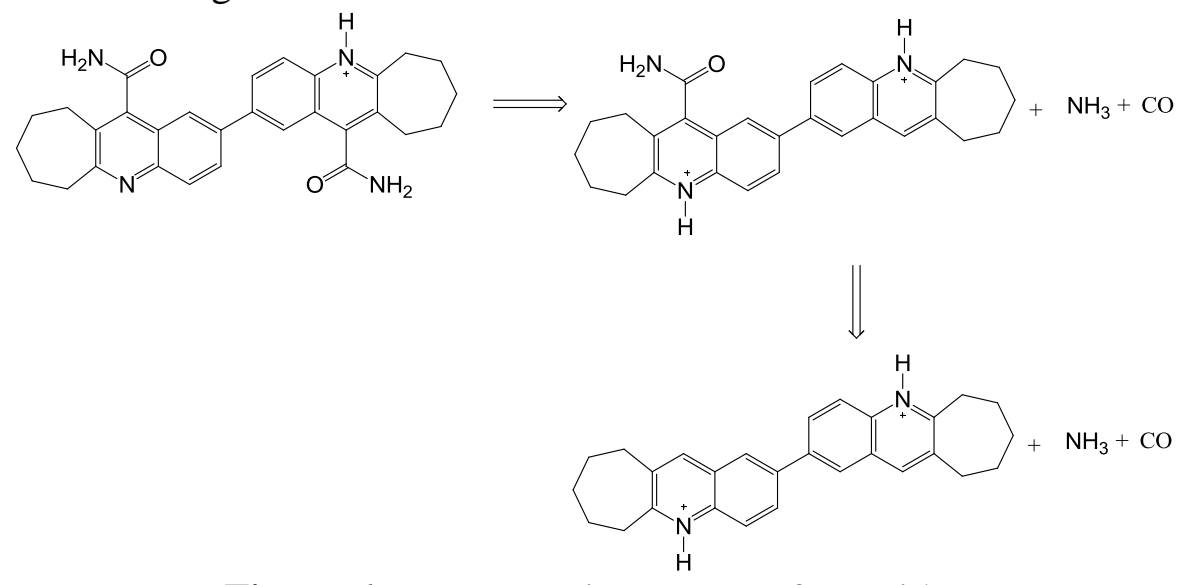

Figure 6. Fragmentation parttern for amides 
Ionization of acids, F7, occurs with the appearance of the double ions $[\mathrm{M}-2 \mathrm{H}]{ }^{2-}$. The acids are ionized also in negative ion mode in the electrospray interface, when a solvent slightly basic is used, such as methanol / water with $0,5 \%$ ammonium carbonate.

The di-acids are strongly ionized in the positive ion mode and fragmentation occurs with difficulty at high collision energies and higher argon pressures $(2.5 \mathrm{mTorr})$. The first fragmentation occurs with the loss of formic acid and the second by the loss of another molecule of $\mathrm{CO}_{2}$ :<smiles>O=C(O)CO</smiles>

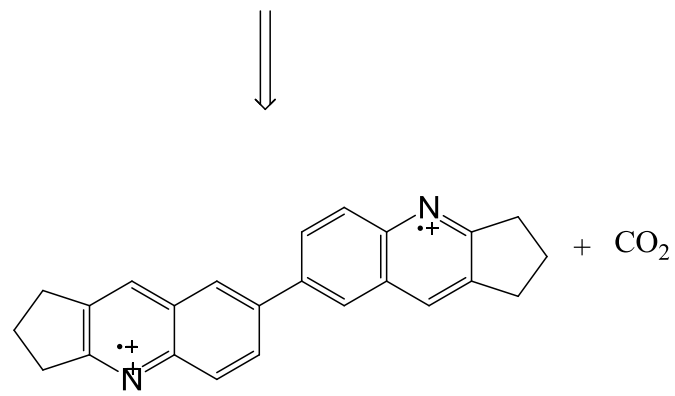

Figure 7. Fragmentation pattern for di-acids

\subsection{Molecular docking studies of compounds with acetylcholinesterase (AChE), butyrylcholine- sterase (BChEI) enzymes}

Table 1 shows the binding free energy of our compounds, as compared with the co-crystallized inhibitors (CCI) and donepezil. The compounds, especially $3 e$ and $5 b$ showed higher binding affinities than the standard drug. $5 b$ showed highest binding affinities of -13.88 and $-15.60 \mathrm{kcal} / \mathrm{mol}$ with $1 \mathrm{OCD}$ and $4 \mathrm{EY} 7$ respectively while $3 e$ had the highest binding affinity with $1 \mathrm{POI}(-13.82 \mathrm{kcal} / \mathrm{mol})$. These compounds seem to have better binding interactions with the drug targets when compared to CCI and donepezil. Consequently, compounds $3 e$ and $5 b$ were used to further illustrate their binding pose and interactions with the targets as shown in figures 8-10. Docking studies of the synthesized compounds into $\mathrm{AChE}$ and $\mathrm{BChEI}$ revealed several interactions between the compounds and the amino acid residues in the binding sites of the drug targets. Figures $6 \mathrm{~A}$ and $\mathrm{B}$ show the binding pose of compound $3 e$ and donepezil respectively in the binding cavity of $\mathrm{BChE}$. Unlike donepezil which had a few significant interactions at the binding cavity of $\mathrm{BChE}, 3 e$ established so many significant interactions with the amino acid residues in the BChE binding site (Figure 8). The amino group of $3 e$ interacted with the oxygen atom of PRO 285 through H-bonding (3.62 $⿱$ A). There were three different $\mathrm{H}$-pi interactions observed with $3 e$ in the binding cavity of BChE. Firstly, the 5-membered ring of TRP 82 interacted with C-26 of the cyclooct-1-enyl of the 3e. Secondly, the 6-membered ring of TYR 332 interacted with the N-atom of the amino group of 3e. Thirdly, 6-membered ring of PHE 329 interacted with the same N-atom as TYR 332 did. CA PRO 285, CE2 PHE 329 and CE2 PHE 329, through pi-H bonding, interacted with two aromatic 6-membered rings and one non-aromatic 6-membered ring of $3 e$ respectively. In Figure 7, compound $3 e$, donezepil and the co-crystallized ligand (butyrate) were concurrently overlaid in the binding cavity of $1 \mathrm{POI}$. These various significant interactions could explain the high binding affinity $3 \mathrm{e}$ had with $\mathrm{BChE}(-13.82 \mathrm{kcal} / \mathrm{mol})$ when compared to the binding affinity of donepezil $(-11.41 \mathrm{kcal} / \mathrm{mol})$ and the crystallized ligand $(-8.02$ 
Revista de Chimie

https://revistadechimie.ro

https://doi.org/10.37358/Rev. Chim.1949

$\mathrm{kcal} / \mathrm{mol}$ ) (Table 1). The few interactions of the crystallized ligand with BChE could possibly account for its weaker BChE inhibitory activity.

Table 1. Binding Free Energy $(\Delta \mathrm{G})$ of synthesized compounds

\begin{tabular}{|c|c|c|c|c|}
\hline & & 1OCD & 4 EY7 & 1P0I \\
\hline $\mathbf{S} / \mathbf{N}$ & Compound & $\Delta \mathrm{G}(\mathrm{kcal} / \mathrm{mol})$ & $\Delta \mathrm{G}(\mathrm{kcal} / \mathrm{mol})$ & $\Delta \mathrm{G}(\mathrm{kcal} / \mathrm{mol})$ \\
\hline 1. & $3 a$ & -12.85 & -15.23 & -12.28 \\
\hline 2. & $3 \mathrm{~b}$ & -12.89 & -14.25 & -12.10 \\
\hline 3. & $3 c$ & -13.23 & -14.55 & -12.67 \\
\hline 4. & $3 d$ & -12.29 & -14.19 & -12.05 \\
\hline 5. & $3 \mathrm{e}$ & -13.32 & -14.65 & -13.82 \\
\hline 6. & $3 \mathrm{f}$ & -12.97 & -14.36 & -12.64 \\
\hline 7. & $5 \mathrm{a}$ & -13.54 & -15.32 & -12.63 \\
\hline 8. & $5 b$ & -13.88 & -15.60 & -13.13 \\
\hline 9 & $\begin{array}{l}\text { Co-crystallized } \\
\text { inhibitor }\end{array}$ & -12.23 & -13.91 & -8.02 \\
\hline 10. & Donepezil & -11.40 & -11.48 & -11.41 \\
\hline
\end{tabular}
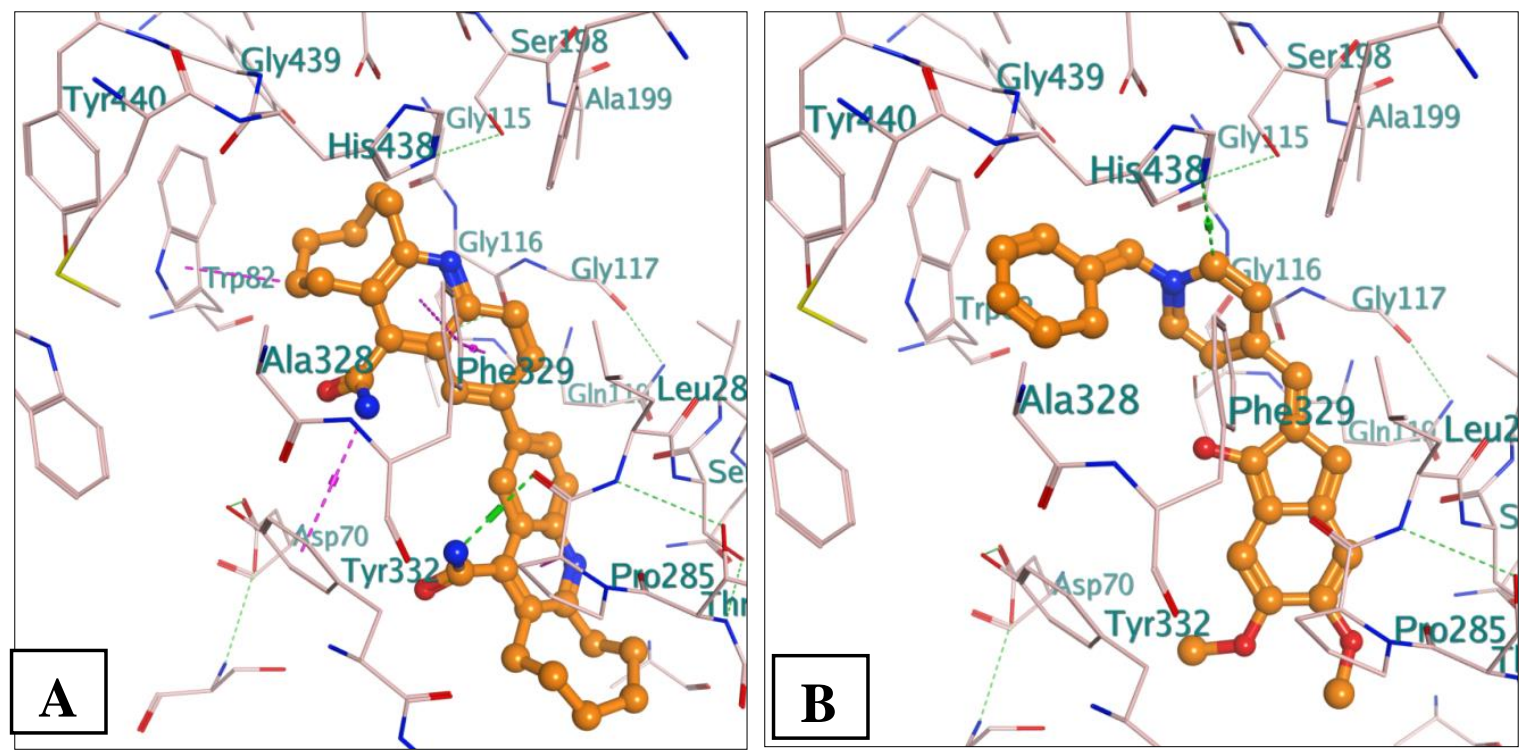

Figure 8. Crystal Structure of human butyrylcholinesterase (1P0I) in complex with (A) compound $3 e$ and $(\mathrm{B})$ donepezil showing their binding poses (green dotted line $=\mathrm{H}$-bond; purple dotted line $=$ pi-H bond 


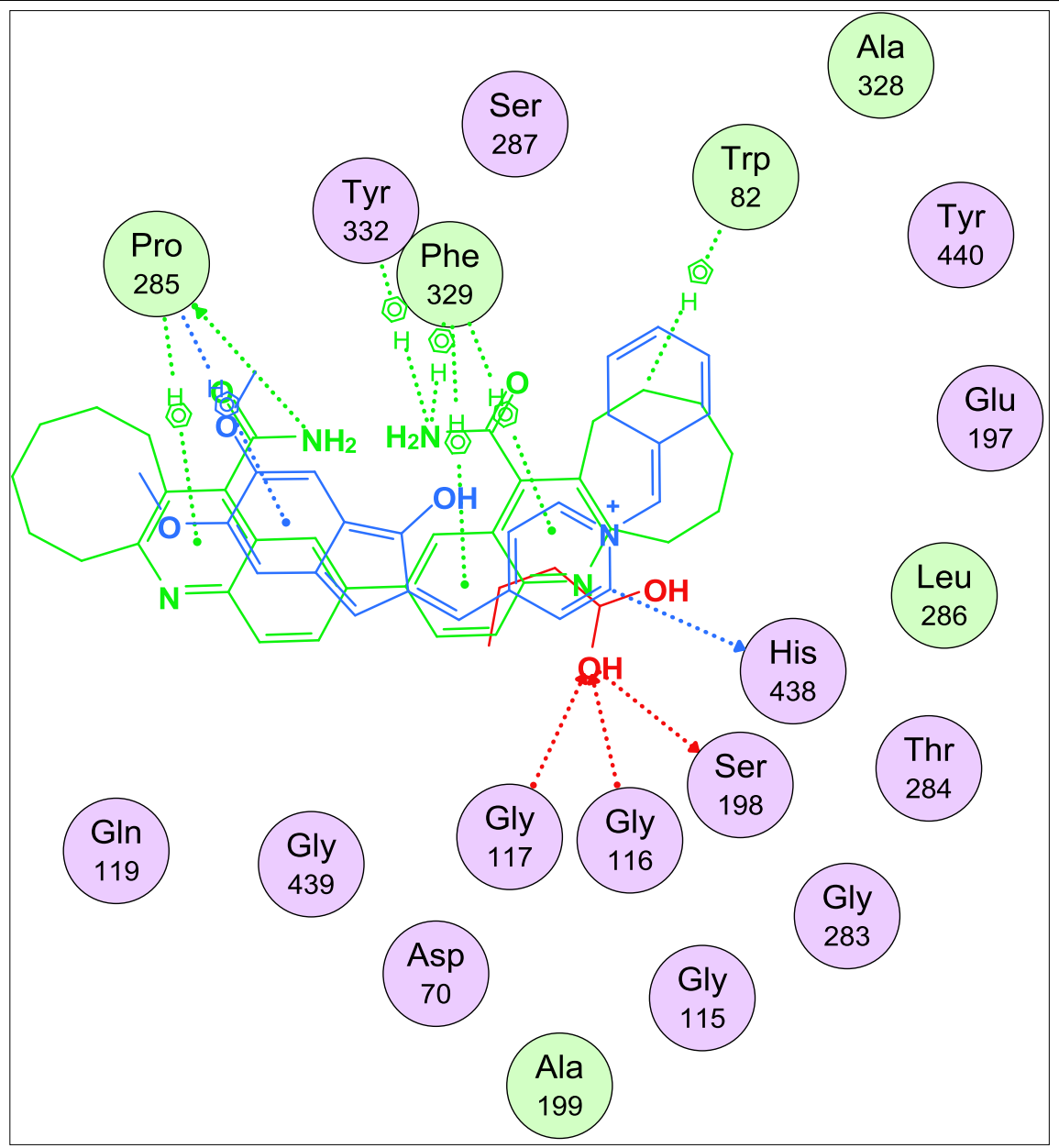

Figure 9. Compound $3 e$ (green) overlaid on the co-crystallized

inhibitor - red (butyrate) and donepezil (blue) in binding site of human butyrylcholinesterase (1P0I)
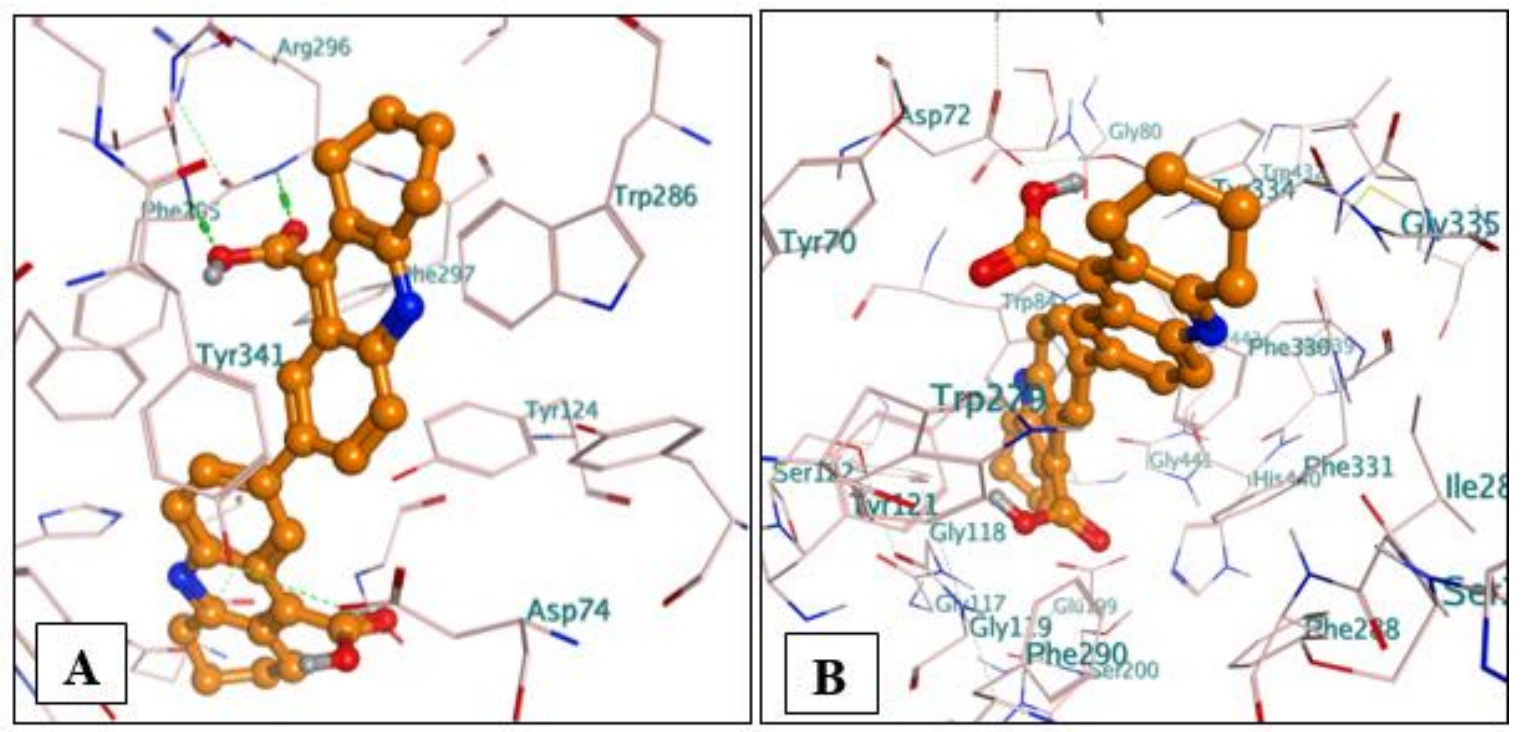

Figure 10. Crystal Structure of acetylcholinesterase (A) 4EY7 and (B) 1OCD in complex compound $5 b$ showing their binding poses (green dotted line $=\mathrm{H}-$ bond; purple dotted line $=$ pi- $\mathrm{H}$ bond 
Figures $10 \mathrm{~A}$ and B showed the binding pose of $5 \mathrm{~b}$ in the binding cavity of 4EY7 and 1OCD of AChE. In Figure 8A, O-33 and O-34 of $5 b$ interacted with N ARG 296 and N PHE 295 through H-acceptor interactions. There was $\mathrm{H}-\pi$ interaction between the $\mathrm{C}-16$ of $5 b$ and 6-membered ring of TRP 86 through an intermolecular distance of $3.75 \AA$. Also, two 6-membered rings of $5 b$ combined with 6-membered rings of TYR 286 and TYR 341 respectively through $\pi-\pi$ interactions. AChE contains two binding site domains namely: catalytic anionic site (CAS) and peripheral anionic site (PAS). The major amino acid residues that constitute PAS include TYR124, TRP286, PHE295, ARG296 and PHE297. It is therefore clear that $5 b$ bound to the PAS of the binding site domain of AChE. PAS, is located at the entrance of the AChE gorge, serves as the binding targets for enzyme inhibitors [32].

\subsubsection{Drug-likeness and pharmacokinetics}

Physicochemical parameters have been used to predict the drug-likeness of a chemical moeity. These parameters influences the pharmacodynamics and pharmacokinetic properties of these chemical compounds. For example, lipophilicity is a property that plays vital role on solubility, absorption, distribution, metabolism, and excretion properties as well as pharmacological activity. When a molecule is highly lipophilic, it tends to go into the inner membrane which is mainly lipoidal and is retained. Octanolwater partition coefficient $(\log \mathrm{P})$ is a parameter that measures the degree of solubility of drugs both in aqueous and lipid phases. The higher the $\log \mathrm{P}$, the higher the chances of the drug molecule to have low solubility. Conversely, when $\log \mathrm{P}$ is lower than the lower limit, the drug will find it difficulty to penetrate the lipid membranes [33]. As a result of higher or lower log P outside the normal range, the drug molecule will have poor bioavailability when administered through oral route. This will directly affect the pharmacological activity of such drug. There are other physicochemical parameters which affect bioavailability of drug molecules and they are shown in Table 2 .

Table 2. Physicochemical parameters for drug-likeness

\begin{tabular}{|c|c|c|c|c|c|c|c|c|c|c|c|c|}
\hline Comp & MW & HBA & HBD & NoRB & $\begin{array}{c}\text { logP } \\
(\mathbf{o} / \mathbf{w})\end{array}$ & TPSA & MR & nA & nAc & \% ABS & LNV & RNV \\
\hline 3a & 450.542 & 4 & 2 & 3 & 4.244 & 111.96 & 13.13 & 60 & 0 & 70.37 & 0 & 1 \\
\hline 3b & 478.596 & 4 & 2 & 3 & 4.960 & 111.96 & 14.01 & 66 & 0 & 70.37 & 0 & 1 \\
\hline 3c & 478.596 & 4 & 2 & 3 & 4.984 & 111.96 & 14.02 & 66 & 0 & 70.37 & 1 & 1 \\
\hline 3d & 478.596 & 4 & 2 & 3 & 5.128 & 111.96 & 14.07 & 66 & 0 & 70.37 & 1 & 1 \\
\hline 3e & 506.650 & 4 & 2 & 3 & 6.012 & 111.96 & 15.00 & 72 & 0 & 70.37 & 2 & 3 \\
\hline 3f & 546.630 & 4 & 2 & 3 & 6.392 & 111.96 & 16.21 & 68 & 0 & 70.37 & 2 & 3 \\
\hline 5a & 424.456 & 6 & 4 & 3 & 4.828 & 100.38 & 11.93 & 52 & 0 & 74.37 & 0 & 1 \\
\hline 5b & 452.510 & 6 & 4 & 3 & 5.712 & 100.38 & 12.87 & 58 & 0 & 74.37 & 1 & 2 \\
\hline
\end{tabular}

MW: molecular weight; HBA: hydrogen bond acceptor; HBD: hydrogen bond donor; TPSA: total polar surface area; nRB: number of rotatable bond; nAc: number of acid; MR: molar refractivity; nA: number of atoms; LNV: Lipinski's number of violations; RNV: Reckitt's number of violation.

These include molecular weight (MW); hydrogen bond acceptor (HBA) - taken as equivalent to the number of oxygen and nitrogen atoms; hydrogen bond donor (HBD) - taken as equivalent to the number of $-\mathrm{OH}$ and $-\mathrm{NH}$ groups; total polar surface area (TPSA); number of rotatable bond (nRB); number of acid (nAc); molar refractivity (AMR) and number of atoms (nA). Lipinski and Reckitt have exploited these physicochemical properties of compounds to predict their drug-likeness. In his rule of five (ro5), 
Lipinski predicted that a drug candidate with good oral bioavailability should have $\mathrm{MW} \leq 500 \mathrm{Da}, \log \mathrm{P} \leq$ $5, \mathrm{HBD} \leq 5$ and HBA $\leq 10$. Reckitt has reported the modified Lipinski's rule of 5 (ro5). He stated that a likely drug molecule should have an $\log \mathrm{P}$ between -0.4 and 5.6, MR between 40 and 130, nA between 20 and 70, $\mathrm{HBD} \leq 5, \mathrm{HBA} \leq 10$ and $\mathrm{MW} \leq 500 \mathrm{Da}$. Any violation of more than one of these physicochemical parameters will be attributed to poor bioavailability and the compound may not be qualified to be a drug candidate.

Our synthesized compounds namely $3 a-d$ and $5 a$ obeyed both the Lipinski's ro5 and the modified Lipinski's ro5 reported by Reckitt. These imply that these compounds can be orally bioavailable and hence are drug candidates. Compounds $5 b$ obeyed only Lipinski's ro5. However, $3 e-f$ disobeyed both rules. However, it should be noted that though some compounds violate these rules, they are still orally bioavailable since they possess groups which act as substrates for biological transporters. They can have up to 4 violations [34]. Furthermore, other surrogate properties have been explored to check whether a compound will be orally bioavailable. A study by [35] has reported that the number of rotatable bond (NoRB) influences bioavailability in rats and recommended that a cut off of NoRB $\leq 10$ show good oral bioavailability. All the synthesized compounds showed NoRB $\leq 10$ indicating a good oral availability. Total polar surface area (TPSA) is another surrogate marker for cell permeability. A molecule with TPSA $\leq 140 \AA^{2}$ would be able to permeate the cell. Again, all the synthesized compounds agreed with this cut off and hence, have the ability to permeate the cell membranes. We further calculated the percentage solubility represented as $\%$ ABS from $\%$ ABS $=109-0.345$ x TPSA [36]. All the synthesized had good solubility at $70.37 \%$ and above which is an indication for good oral bioavailability

$\mathrm{AD}$ is a neurological disorder. For any drug to be considered as an effective treatment for AD, it must essentially cross the BBB to gain access to the brain cells. This is of primary consideration in the design of drugs for neurological intervention. Besides crossing the $\mathrm{BBB}$, a potential neurotherapeutic drug candidate would need to stay in the brain tissue for a reasonable time in order to elicit the expected pharmacological action [37, 38]. Andreas Reichel has modified the Lipinski's ro5 for neurotherapeutics to 'CNS likeness' [39]. He stated that a neurotherapeutic must have MW $<450 \mathrm{Da}, \log \mathrm{P}<3, \mathrm{HBD}<4$ and $\mathrm{HBA}<8$. However, Reichel failed to take into consideration other essential drug properties such as polar surface area, number of rotatable bonds, $\mathrm{pKa}$, flexibility, and $\log \mathrm{D}$ at $p \mathrm{H} 7.4(\log \mathrm{D} 7.4)$ which are desirable for BBB permeation. These drug properties are shown in Table 3 and are modifications from [40, 41].

Table 3. Benchmark criteria for CNS-likeness of neuropharmaceutical

\begin{tabular}{cc}
\hline Property & The cut-off for BBB penetrability \\
\hline Molecular weight & $400-500 \mathrm{Da}$ \\
Log P & $<7$ \\
Polar surface area & $60-70 \AA$ \\
Hydrogen dond donor & $<3$ \\
Hydrogen bond acceptor & $<7$ \\
Number of rotatable bond & $<8$ \\
Number of acid & 0 \\
\hline
\end{tabular}

Apart from the violation of the TPSA which is inherent in all the compounds, most of the synthesized compounds met the requirement/cut-off for BBB penetrability. This implies that they can cross the blood brain barrier and might be useful to treat AD.

\subsubsection{Pharmacokinetic assessment}

Only unbounded drug will be available for diffusion and transport across cell membranes. This will ultimately be available to interact with the pharmacological targets. Therefore, the extent of plasma protein binding of drug molecules will determine its therapeutic outcome, distribution and duration in the 
system. It has been found out that drug molecules with PPB of more than $90 \%$ are strongly bound to the plasma protein and those with less than $90 \%$ are weakly bound. From table 4 , it can be seen that all the synthesized compounds are strongly bound to plasma protein except $3 d$. Drugs that are highly bound to plasma protein will be less readily available in the systemic circulation. However, they can be formulated as sustained release drugs.

Caco-2 model and Human Intestinal Absorption (HIA) are useful in assessing the cell permeability and intestinal absorption of drug molecule. Caco-2 values being less than 4 imply low permeability; 4 - 70 implies middle permeability and greater than 70 implies high permeability [44]. Yee [43] collaborated this finding in 1999, by showing that compounds with HIA (0 -20\%) indicates poor absorption; 20 - 70\% are moderately absorbed and $(70-100 \%)$ are well absorbed. Our compounds in table 4 show moderate permeability and they can be well absorbed in the intestine.

CYP2C19 is a member of the cytochrome P450 superfamily of drug-metabolizing enzymes involved in the metabolism of the majority of currently prescribed drugs and new chemical entities. It is a polymorphic enzyme that accounts for less than 5\% of hepatic P450 [44] and 2 to 3\% of intestinal P450 content [45]. All the synthesized compounds are inhibitors of CYP2C19. That implies that drugs which are metabolized by this enzyme should not be co-administered with these compounds if formulated as drugs. Example: proton pump inhibitors such as omeprazole and lansoprazole, psychotropic drugs including diazepam and imipramine, and anticonvulsants such as phenobarbital and mephenytoin are metabolized by CYP2C19.

Table 4. Pharmacokinetics parameters

\begin{tabular}{|c|c|c|c|c|c|}
\hline Comp & BBB & Caco-2 & HIA & PPB & $\begin{array}{l}\text { CYP 2C19 } \\
\text { Inhibition }\end{array}$ \\
\hline $3 a$ & 0.02123 & 20.687 & 95.84 & 90.38 & Inhibitor \\
\hline $3 b$ & 0.03222 & 20.81 & 95.99 & 90.13 & Inhibitor \\
\hline $3 c$ & 0.05040 & 20.78 & 95.99 & 92.64 & Inhibitor \\
\hline $3 d$ & 0.04967 & 20.74 & 95.99 & 88.38 & Inhibitor \\
\hline $3 e$ & 0.18485 & 20.83 & 96.18 & 91.17 & Inhibitor \\
\hline $3 \mathrm{f}$ & 0.03741 & 20.82 & 97.31 & 95.83 & Inhibitor \\
\hline $5 a$ & 0.02344 & 20.87 & 97.10 & 94.98 & Inhibitor \\
\hline $5 \mathrm{~b}$ & 0.02917 & 20.92 & 96.95 & 96.04 & Inhibitor \\
\hline
\end{tabular}

\section{Conclusions}

The Bis-polymethylenquinoline-bis-carboxamides can be considered dual-binding site heterodimers in which the two tetrahydroacridine units are connected directly through the benzenic nucleus of tetrahydroacridinic skeleton. In this present work, a series of di-polymethylencarboxamides and the corresponding diacids were designed to simultaneously interact with the active and peripheral binding sites of human AChE. The compounds where obtained by Pizinger condensation reaction of precursors 5,5'- 5,5'-biindoline-2,2',3,3'-tetraone, 2a with substituted cyclanones. The carboxamides are obtained with a yield of $40-50 \%$. Opposed to the mono tetrahydroacridines, the new dimers had very high melting points (mostly decomposed above $240^{\circ} \mathrm{C}$ ) and are insoluble in common organic solvents. The pharmacological potentials of the compounds, in terms of binding modes, as inhibitors of human acetylcholinesterase (hAChE) and butyrylcholinesterase (hBChE) were evaluated by molecular docking studies with the bio-target sites of AChE: 4EY7, 1OCD; BChE: 1P0I. The compounds had good interactions with the biotargets. However $3 e$ and $5 d$ showed the best binding (greater than the cocrystallized inhibitors and donezepil). These compounds could become lead in the treatment of Alzheimer's disease. 


\section{References}

1.APOStOlOVA L. G., Alzheimer Disease, Continuum (Minneap Minn). 2016 , p. 419, doi: 10.1212/CON.0000000000000307.

2. BURNS A., Alzheimer's disease, 2009, p.338:b158, https:// doi.org/ 10.1136/ bmj.

3.a) J. HUGO, M. GANGULI, Clin. Geriatr. Med., 30, nr.3, 2014, p. 421, doi: 10.1016/j.cger. 2014.04.001; b)Alistair Burns, Dementia, 2009; p.338, b75, doi: https://doi.org/10.1136/bmj.b75.

4. SADOWSKI, M., WISNIEWSKI, T., Curr. Pharm. Des. 13, 2007, p. 1943.

5. PANEK, D., WICHUR, T., GODYŃ, J., PASIEKA, A., MALAWSKA, B. , Future Med. Chem., 9, nr.15, 2017, p. 1835. doi:10.4155/fmc-2017-0094.

6. AlvareZ, A., OPAZO, C., AlARCON, R., GARRIDO, J., INESTROSA, N.C., J. Mol. Biol. , 272,1997, p.348.

7. COYLE J.T., PUTTFARCKEN P.,Science. 262, nr. 5134, 1993, p.689.

8. MYHRE, O., UTKILEN, H., DUALE, N., BRUNBORG, G., , HOFER, T., Oxid. Med. and Cell. Longev., 2013, Article ID 726954, http://dx.doi.org/10.1155/2013/726954.

9. GREIG UTSUKI, T., INGRAM, D.K., WANG, Y., PEPEU, G., C.SCALIYU, Q.S., MAMCZARZ, J., HOLlOWAY, H.W., GIORDANO, T., CHEN, D., FURUKAWA, K., SAMBAMURTI, K., BROSSI, A., LAHIRI, D.K., Proc. Natl. Acad. Sci. , 102, 2005, p. 17213.

10. TERRY, A.V., BUCCAFUSCO, J.J., J. Pharmacol. Exp. Ther., 306, 2003, p. 821.

11. LANE, R. M., POTKIN, S. G., ENZ, A. , Int. J. Neuropsychopharmacol., 9, nr. 1, 2005, 101. doi:10.1017/s1461145705005833

12. NICOLET Y., LOCKRIDGE O., MASSON P., FONTECILLA-CAMPS J.C., NACHON F., J. Biol. Chem., 278, nr.42, 2003, p. 41141.

13. PODOLY, E., SHALEV, D.E., SHENHAR-TSARFATY, S., BENNETT, E.R., BEN ASSAYAG, E., WILGUS, H., LIVNAH, O., SOREQ, H., J. Biol. Chem., 284, 2009, p.17170.

14. BARTOLINI, M., BERTUCCI, C., CAVRINI, V., ANDRISANO, V., Biochem. Pharmacol., 65, 2003, p. 407.

15. INESTROSA, N.C., AlVAREZ, A., PÉREZ, C.A., MORENO, R.D., VICENTE, M., LINKER, C., CASANUEVA, O.I., SOTO, C., GARRIDO, J., Neuron., 16, nr.4,1996 , p. 881.

16. ANAND P., SINGH B. , Arch. Pharm. Res., 36, 2013, p. 375-399.

17. BALlARD, C.G., GREIG, N.H., GUILlOZET-BONGAARTS, A.L., ENZ, A., DARVESH, S., Curr. Alzheimer. Res., 2, 2005, p.307.

18. PROCTOR, G.R., HARVEY, A.L., Curr.Med. Chem.,7, 2000, p. 295.

19.a) CRISMON, M. L., Annals of Pharmacotherapy, 28, nr. 6, 1994, p. 744. doi:10.1177/106002809402800612 . b.) QIZILBASH, N., BIRKS, J., LÓPEZ ARRIETA, J., , S., SZETO, S.Cochrane Database of Systematic Reviews 1999, Issue 1. Art. No.: CD000202. DOI: 10.1002/14651858. CD000202.

20. LAGADIC-GOSSMANN,D., RISSEL, M., LE BOT, M.A.,GUILLOUZO, A., Cell Biol. Toxicol. 14, 1998, p.361.

21. MUNOZ-TORRERO, D., CAMPS, P., Curr. Med. Chem. , 13, 2006, p.399.

22. BAUTISTA AGUILERA, O., ESTEBAN, G., CHIOUA, M., NIKOLIC, K., AGBABA, D, MORALEDA, I., IRIEPA, I., SORIANO, E., SAMADI, A., UNZETA, M., MARCO-CONTELLES, J., Drug Des. Devel. Ther., 8, 2014, p.1893.

23. BAJDA, M., WIĘCKOWSKA, A., HEBDA, M., GUZIOR, N., SOTRIFFER, C., \& MALAWSKA, B., Int. J. Mol. Sci., 14, nr.3, 2013, p.5608. doi:10.3390/ijms14035608.

24. GUZIOR, N., WICKOWSKA, A., PANEK, D., MALAWSKA, B., Curr. Med. Chem., 22, 2015, p.373. 
25. HRUBARU M.M., MAGANU, M., VUlPES, D., AlEXANDRU, M.G., PlAVETI, M., Rev. Chim, 60, (9), 2009, p.859.

26. HRUBARU, M.M., TARKO, L., Rev. Chim., 70, (3), 2019, p. 887.

27. HRUBARU, M.M., TARKO, L., Rev. Chim., 70, (8), 2019, p.3077.

28. MENON, K. N.; RAMAN, P. S., Proceedings of the Indian Academy of Sciences, Section A, 38,nr. 2 , 1953 , p.132. http://dx.doi.org/10.1007/BF03045213.

29. BIELAVSKY, J., Coll. Czech. Chem.Chim. Commun., 42, 1977, p.2802.

30.NICOLET,Y., LOCKRIDGE,O., MASSON,P., FONTECILLA-CAMPS， J.C., NACHON， F. ， J. Biol.Chem., 278, 2003, p. 41141. DOI: 10.1074/jbc.M210241200.

31. SZYMANSKI, P., KARPIŃSKI, A., MIKICIUK-OLASIK, E., Eur. J. Med. Chem., 46, nr. 8, 201 Oxid Med Cell Longev 1, p.3250.

32. SUSSMAN J.L., HAREL M., FROLOW F., OEFNER C., GOLDMAN A., TOKER L., SILMAN I. Atomic structure of acetylcholinesterase from Torpedo californica: a prototypic acetylcholine-binding protein, Science. 253, nr.5022, 1991, p.872.

33.MCNALLY, B. A., LEEVY, W. M., \& SMITH, B. D, 19, nr.1-2, 2007, p.29, DOI: 10.1080/ 10610270600902332.

34. PETIT, J., MEURICE, N., KAISER, C., MAGGIORA, G., Softening the Rule of Five-where to draw the line?, Bioorg. Med. Chem., 20, nr. 18, 2012, p. 5343; https://doi.org/10.1016/j.bmc.2011.11.064

35. VEBER D.F., JOHNSON S.R., CHENG H.Y., SMITH B.R., WARD K.W., KOPPLE K.D., J. Med. Chem., 45, nr.12, 2002, p.2615; DOI: 10.1021/jm020017n.

36. ZHAO Q., WANG J., LEVICHKIN I.V., STASINOPOULOS S., RYAN M.T., HOOGENRAAD N.J., EMBO J., 21, nr.17, 2002, p.4411.

37. BEGLEY D.J., Pharmacol Ther. 104, nr.1, 2004 , p. 29.

38. LÖSCHER W., POTSCHKA H., NeuroRx., 2, nr.1, 2005, p.86; DOI: 10.1602/neurorx.2.1.86

39. REICHEL A., Curr. Drug Metab., 7, nr.2, 2006, p.183, DOI: 10.2174/138920006775541525.

40. JOUYBAN A., SOLTANI S., Toxicity and Drug Testing, William Acree, IntechOpen, DOI: 10.5772/20360. Available from: https://www.intechopen.com/books/toxicity-and-drug-testing/bloodbrain-barrier-penetration.

41. UGWU, D. I., OKORO, U. C., UKOHA, P. O., GUPTA, A., OKAFOR, S. N., J. Enzyme Inhib. Med. Chem., 33, nr.1, 2018, p.405, DOI: 10.1080/14756366.2018.1426573.

42. YAMASHITA S., FURUBAYASHI T., KATAOKA M., SAKANE T., SEZAKI H., TOKUDA H., Eur. J. Pharm. Sci., 10, nr.3, 2000, p.195.

43. YEE S, Pharm. Res., 14, nr. 6, 1997, p.763.

44. RING B. J., GILLESPIE J. S., ECKSTEIN J. A., WRIGHTON S. A., Drug Metab. Dispos., 30, nr.3, 2002, p.319. DOI: https://doi.org/10.1124/dmd.30.3.319 .

45. PAINE M.F., HART H.L., LUDINGTON S.S., HAINING R.L., RETTIE A.E., ZELDIN D.C., Drug. Metab. Dispos., 34, nr.5, 2006 , p.880.

Manuscript received: 30.08 .2019 